\title{
Response of Duffing's Oscillator to Harmonic Base Excitation and Significance of First Order Term
}

\author{
K. Divya \\ Structures Group, UR Rao Satellite center, ISRO Vimanapura Post, Bangalore, India 560017.
}

\section{K. Renji}

Advanced Technology Development Group, UR Rao Satellite center, ISRO Vimanapura Post, Bangalore, India 560017.

\section{(Received 9 November 2019; accepted 1 April 2020)}

Responses of systems with nonlinear stiffness subjected to base harmonic excitation are determined. An expression to estimate the amplitude in the fundamental frequency of oscillation is derived from first principles using Lindstedt's method. It is observed that the amplitude determined using the zero order approximation is in error at low frequencies. Therefore, an expression for the first order approximation of the amplitude of response at the fundamental frequency is derived. Zero order and first order approximation terms together form the response. Characteristics showing the variation of the amplitude with the excitation frequency for various nonlinear spring parameters are presented. The issue at low frequencies is resolved by the incorporation of the first order term. An expression for the phase difference and the expression of the asymptote where the responses converge are also derived.

\section{NOMENCLATURE}

\section{$k \quad$ stiffness}

m mass

c coefficient of viscous damping

$f_{\text {spring }}$ spring restoring force

$x \quad$ absolute displacement of the system

$\dot{x} \quad$ velocity of the system

$\ddot{x} \quad$ acceleration of the system

$\epsilon \quad$ small parameter, $\epsilon<<1$

$\alpha, \beta \quad$ parameters related to the nonlinear spring

$\epsilon \zeta \quad$ damping factor

$\omega \quad$ parameter related to the natural frequency

$y \quad$ base displacement

$\epsilon Y \quad$ amplitude of the base excitation

$\Omega \quad$ frequency of excitation

$t \quad$ time

$T \quad$ time period of oscillation

$\tau \quad$ modified time variable

$\varphi \quad$ phase angle

$\omega_{0} \quad$ linearised natural frequency

$x_{i} \quad i^{t h}$ order term of the displacement, $i=0,1,2 \ldots$

$\varphi_{i} \quad i^{t h}$ order term of the phase angle, $i=0,1,2 \ldots$

$A_{i} \quad i^{\text {th }}$ order term of the amplitude of the displacement, $i=0,1,2 \ldots$

\section{INTRODUCTION}

Many systems encountered in practice exhibit nonlinear behavior $^{1,2}$ due to the change in their stiffness with deformation. This may be because of its geometrical construction or the characteristics of the material itself. Bi-linear and cubic are a couple of simple models that are used for representing such stiffness characteristics. A single degree-of-freedom (SDOF) system with a spring having cubic non-linearity is called a
Duffing's oscillator. Many equipment, machines, buildings etc. are provided with isolators to reduce their vibrations or the vibrations transmitted. Behavior of all such isolators can be represented by this model. Vaiana et. al., ${ }^{3}$ Sudhir Kaul, ${ }^{4}$ Losanno et. al. ${ }^{5}$ and Madera Sierra et. al. ${ }^{6}$ used such models to represent the behavior of isolators, which show that such models are generally used for representing the nonlinear stiffness of isolators. These models find place even in the analysis of non-linear dampers $^{7-9}$ and other devices. ${ }^{10}$

Determining the response of a Duffing's oscillator is a classic example of a nonlinear problem and a large amount of work is carried out on this subject. The text book by Meirovitch ${ }^{11}$ gives a good insight into this problem. Considering the nonlinearity to be weak, perturbation techniques are widely used for arriving at the solution. Lindstedt's method, method of averaging, harmonic balance method, method of multiple scales are the most commonly used perturbation techniques. ${ }^{12,13}$

Using the above mentioned techniques, the free vibration characteristics and the response to harmonic force excitation are studied extensively. These studies have provided expressions for determining the frequency of the free vibration of the Duffing's oscillator. Expressions were also presented for the amplitude of the response when excited by a harmonic force. ${ }^{11}$ The responses show 'jump phenomenon' when they are subjected to force excitation. There are several works that discuss on the jump phenomenon. ${ }^{14-18}$ Malatkar and Nayfeh ${ }^{16}$ obtained the minimum force that needs to be applied to make the system to have jumps in their responses. Brennan et.al. ${ }^{17}$ obtained expressions for determining the frequencies at which the jumps in the responses occur. Works are also reported in determining the parameters of the nonlinear system from its response characteristics using methods like nonlinear subspace identification ${ }^{18}$ etc. All these works are related to force excitation. 
Many of such nonlinear systems exhibit hysteresis. A suitable model that represents this type of hysteresis is Bouc-Wen model. This model was proposed initially by Bouc ${ }^{19}$ and later generalized by Wen. ${ }^{20,21}$ In this model the restoring force is related to the displacement through a first order nonlinear differential equation. By assigning suitable values for the parameters, the required hysteresis loop is represented. Vaiana et. al. ${ }^{22-24}$ developed several models that provide significant computational advantages while representing the hysteresis behavior.

Though there are several works carried out on the response of nonlinear systems when subjected to force excitation as well as complex models that represent their hysteresis behavior, it is seen that the works reported on the response to base excitation are very few. Hundal ${ }^{25}$ determined the response of systems to base excitation with nonlinear damping. Xiaojuan Sun and Jianrun Zhang ${ }^{26}$ worked on the response of isolators with nonlinear damping. In these works, the stiffness was considered to be linear. Responses are also obtained for modulated sinusoidal excitation using method of multiple scales, ${ }^{27}$ but it was for a force excitation and not for a base excitation. In an another study ${ }^{28}$ the response to base excitation of an oscillator is determined with a nonlinearity in the secondary spring. The main spring was still linear and the nonlinear spring connects only the damper.

Ravindra and Mallik ${ }^{29,30}$ obtained the response of a nonlinear system when subjected to base excitation. They used harmonic balance method for determining the solution. Zarko Milovanovic et.al. ${ }^{31}$ derived an expression for determining the amplitude of the response of a vibration isolator subjected to base excitation. Bahareh Zaghari et.al. ${ }^{32}$ determined the response to base excitation along with force excitation. In all the above works ${ }^{29-32}$ the base excitation problem is written in the form of force excitation problems and the relative responses are determined using the expressions for force excitation. The absolute displacements are then determined from the relative displacements. While doing the above, the phase of the absolute displacement is assumed, thus it does not give an analytically correct solution. Other works reported on base excitation $^{33-35}$ adopt numerical techniques to determine the solution and hence no expressions are derived. A few works reported $^{36,37}$ on response to base excitation, deal with cantilevered beam with tip mass. In those works, numerical techniques are used to determine the solution and no expressions are derived. Also, the nonlinearities present in those investigations are not of the cubic type.

Though there are expressions reported for determining the amplitude of the response of Duffing's oscillator to base sine excitation, ${ }^{29-32}$ they are derived with certain assumptions on the phase difference which is analytically not exact. In this work, the absolute response is derived from the first principles (not from the relative response) without any such assumption. Here, Lindstedt's perturbation technique which is mathematically rigorous is chosen to derive the expression for the amplitude. As in the previous studies the nonlinearity is assumed to be small. In previous works, only the zero order approximation was considered. It is shown that the first order approximation terms are also essential and expressions for these terms are derived. Using these expressions, characteristic curves for the

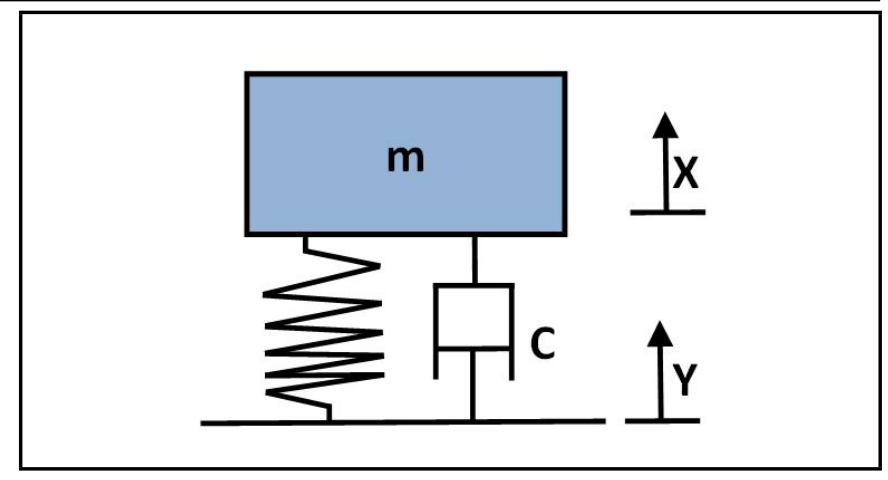

Figure 1. Duffing's oscillator with base excitation.

base sine excitation are obtained. These results are particularly useful for Isolators used in sensitive equipment / optical elements mounted in spacecraft / aircraft, isolators used in gyros, isolators in building etc.

\section{ZERO ORDER APPOXIMATION OF RESPONSE}

Lindstedt's method well established for determining the response to force excitation ${ }^{3}$ is used to obtain the response to base excitation.

Let us consider a quasi-harmonic system as shown in Fig. 1 subjected to a base harmonic excitation. It consists of a mass, a spring and a damper.

\subsection{Equation of Motion}

The spring force is given by ${ }^{11}$ :

$$
f_{\text {spring }}(x)=k x+\epsilon k\left(\alpha x+\beta x^{3}\right) ;
$$

where $x$ is the absolute displacement. The first derivative of $f_{\text {spring }}(x)$ with respect to the displacement gives the stiffness of the spring and in general it is a function of the displacement. If $\beta$ is $+v e$, stiffness increases with the displacement. Such springs are called hardening spring. In a softening spring the stiffness reduces with the displacement and $\beta$ is - ve. If $\beta=$ 0 , the system will be linear and the corresponding stiffness is $k(1+\epsilon \alpha)$.

The second-order ordinary differential equation of motion of a linear SDOF system having mass $m$, damping coefficient $c$ and stiffness $k$, when subjected to a base displacement $y$, is:

$$
m \ddot{x}+c \dot{x}+k x=c \dot{y}+k y .
$$

When the spring force is as given in Eq. (1), the differential equation of motion becomes:

$$
\begin{aligned}
& m \ddot{x}+c \dot{x}+k(1+\epsilon \alpha) x+\epsilon k \beta x^{3}= \\
& \quad c \dot{y}+k(1+\epsilon \alpha) y+\epsilon k \beta y^{3} .
\end{aligned}
$$

The right hand side of the above equation has several terms with one nonlinear term whereas in the case of force excitation there is only one term and it is linear. Dividing Eq. (3) by the mass, we get:

$$
\begin{aligned}
\ddot{x}+\omega^{2} x=\epsilon\left[-\omega^{2}\right. & \left.\left(\alpha x+\beta x^{3}\right)-2 \zeta \omega \dot{x}\right] \\
+ & 2 \epsilon \zeta \omega \dot{y}+\omega^{2} y+\epsilon \omega^{2}\left(\alpha y+\beta y^{3}\right) ;
\end{aligned}
$$


where, $\omega^{2}=k / m$ and $\epsilon \zeta=c /(2 m \omega)$ is the damping factor.

For a harmonic base excitation of $y=\epsilon Y \cos \omega t$, the equation of motion in terms of absolute displacement can be shown to be:

$$
\begin{aligned}
\ddot{x}+\omega^{2} x= & \epsilon\left[-\omega^{2}\left(\alpha x+\beta x^{3}\right)-2 \zeta \omega \dot{x}\right] \\
-2 \epsilon & \zeta \omega \Omega \epsilon Y \sin \Omega t+\omega^{2} \epsilon Y \cos \Omega t \\
& \quad+\epsilon \omega^{2}\left(\epsilon \alpha Y \cos \Omega t+\beta \epsilon^{3} Y^{3} \cos ^{3} \Omega t\right) .
\end{aligned}
$$

Equation (5) represents the differential equation of motion of the Duffing's oscillator when subjected to base excitation.

\subsection{Expression for Amplitude of the Absolute Displacement}

Our aim is to obtain a periodic solution of Eq. (5). The period of excitation is $T=\frac{2 \pi}{\Omega}$. Shifting the time scale $t$ to $\tau$, which is the new time variable so that the period of oscillation is $2 \pi$ (and not $\frac{2 \pi}{\Omega}$ ), the equation of motion changes to:

$$
\begin{aligned}
\Omega^{2} x^{\prime \prime} & +\omega^{2} x=\epsilon\left[-\omega^{2}\left(\alpha x+\beta x^{3}\right)-2 \zeta \omega \Omega x^{\prime}\right] \\
& -2 \epsilon \zeta \omega \Omega \epsilon Y \sin (\tau+\varphi)+\omega^{2} \epsilon Y \cos (\tau+\varphi) \\
& +\epsilon \omega^{2}\left[\epsilon \alpha Y \cos (\tau+\varphi)+\beta \epsilon^{3} Y^{3} \cos ^{3}(\tau+\varphi)\right]
\end{aligned}
$$

where $\varphi$ is the phase angle. While doing so we employed the transformations $\tau=\Omega t-\varphi$ and $\frac{d}{d t}=\Omega \frac{d}{d \tau}$.

For a solution as given below:

$$
\begin{gathered}
x=x_{0}+\epsilon x_{1}+\epsilon^{2} x_{2}+\ldots ; \\
\varphi=\varphi_{0}+\epsilon \varphi_{1}+\epsilon^{2} \varphi_{2}+\ldots ;
\end{gathered}
$$

the equation of motion becomes (taking $x=x_{0}+\epsilon x_{1}$ ):

$$
\begin{aligned}
& \Omega^{2}\left(x_{0}+\epsilon x_{1}\right)^{\prime \prime}+\omega^{2}\left(x_{0}+\epsilon x_{1}\right)= \\
& \epsilon\left[-\omega^{2}\left(\alpha\left[x_{0}+\epsilon x_{1}\right]+\beta\left[x_{0}+\epsilon x_{1}\right]^{3}\right)-2 \zeta \omega \Omega\left(x_{0}+\epsilon x_{1}\right)^{\prime}\right] \\
& \quad-2 \epsilon \zeta \omega \Omega \epsilon Y \sin (\tau+\varphi)+\omega^{2} \epsilon Y \cos (\tau+\varphi) \\
& \quad+\epsilon \omega^{2}\left[\epsilon \alpha Y \cos (\tau+\varphi)+\beta \epsilon^{3} Y^{3} \cos ^{3}(\tau+\varphi)\right] .
\end{aligned}
$$

This can be further written as:

$$
\begin{aligned}
& \left(\Omega^{2} x_{0}^{\prime \prime}+\omega^{2} x_{0}\right)+\epsilon\left(\Omega^{2} x_{1}^{\prime \prime}+\omega^{2} x_{1}\right)= \\
& \epsilon\left[-\omega^{2}\left(\alpha x_{0}+\beta x_{0}^{3}\right)-2 \zeta \omega \Omega x_{0}^{\prime}+\omega^{2} Y \cos (\tau+\varphi)\right] \\
& \quad+\epsilon^{2}\left[-\omega^{2}\left(\alpha x_{1}+3 \beta x_{0}^{2} x_{1}\right)-2 \zeta \omega \Omega x_{1}^{\prime}+\right. \\
& \left.2 \zeta \omega \Omega Y \sin (\tau+\varphi)+\omega^{2} \alpha Y \cos (\tau+\varphi)\right] \\
& +\epsilon^{3}\left[3 \beta x_{0} x_{1}^{2}+\beta Y^{3} \cos ^{3}(\tau+\varphi)\right]+\epsilon^{4} \beta x_{1}^{3} .
\end{aligned}
$$

It can be shown that with $\cos \left(\epsilon \varphi_{1}\right)=1$ and $\sin \left(\epsilon \varphi_{1}\right)=\epsilon \varphi_{1}$ for small values of $\epsilon$ (for $\epsilon \varphi_{1}<0.3$, the error in the trigonometric terms will be less than $5 \%$ ):

$$
\begin{aligned}
\cos (\tau+\varphi)= & \cos \tau \cos \varphi_{0}-\sin \tau \sin \varphi_{0} \\
& -\epsilon \varphi_{1}\left\{\cos \tau \sin \varphi_{0}+\sin \tau \cos \varphi_{0}\right\}
\end{aligned}
$$

$$
\begin{aligned}
\sin (\tau+\varphi)= & \sin \tau \cos \varphi_{0}+\cos \tau \sin \varphi_{0} \\
& -\epsilon \varphi_{1}\left\{\sin \tau \sin \varphi_{0}-\cos \tau \cos \varphi_{0}\right\} .
\end{aligned}
$$

Using the above relations in Eq. (10) and equating the coefficients of terms having the same powers of $\epsilon$, we get:

$$
\Omega^{2} x_{0}^{\prime \prime}+\omega^{2} x_{0}=0
$$

$$
\begin{aligned}
\Omega^{2} x_{1}^{\prime \prime}+\omega^{2} x_{1}= & -2 \zeta \omega \Omega x_{0}^{\prime}-\omega^{2}\left(\alpha x_{0}+\beta x_{0}^{3}\right) \\
& +\omega^{2} Y\left\{\cos \tau \cos \varphi_{0}-\sin \tau \sin \varphi_{0}\right\}
\end{aligned}
$$

The $x_{i}(\tau), i=0,1,2, \ldots$ are solved sequentially, subject to the periodicity condition $x_{i}(\tau+2 \pi)=x_{i}(\tau)$ and the initial conditions $x_{i}^{\prime}(0)=0$.

The solution of the first equation is:

$$
x_{0}=A_{0} \cos \left(\frac{\omega}{\Omega}\right) \tau .
$$

To have a periodic solution, $\omega=\Omega$ and hence the zero order solution becomes:

$$
x_{0}=A_{0} \cos \tau .
$$

$A_{0}$ is determined from the second differential equation, i.e., Eq. (14).

$$
\begin{aligned}
& x_{1}^{\prime \prime}+x_{1}=2 \zeta A_{0} \sin \tau \\
& -\left(\alpha A_{0} \cos \tau+\beta\left(A_{0} \cos \tau\right)^{3}\right)+Y \cos \left(\tau+\varphi_{0}\right) .
\end{aligned}
$$

Recalling that $\cos ^{3} \tau=\frac{1}{4}(3 \cos \tau+\cos 3 \tau)$ we get:

$$
\begin{aligned}
& x_{1}^{\prime \prime}+x_{1}=\left(2 \zeta A_{0}-Y \sin \varphi_{0}\right) \sin \tau \\
& +\left(-\alpha A_{0}-\frac{3}{4} \beta A_{0}^{3}+Y \cos \varphi_{0}\right) \cos \tau-\frac{1}{4} \beta A_{0}^{3} \cos 3 \tau .
\end{aligned}
$$

The solution must satisfy the periodicity condition to prevent the occurrence of secular term. ${ }^{11}$ Therefore, coefficients of $\sin \tau$ and $\cos \tau$ should vanish. This leads to:

$$
\begin{gathered}
2 \zeta A_{0}=Y \sin \varphi_{0} ; \\
\alpha A_{0}+\frac{3}{4} \beta A_{0}^{3}=Y \cos \varphi_{0} .
\end{gathered}
$$

To determine $A_{0}$, multiply Eqs. (19) and (20) by $\varepsilon$. Squaring both sides of Eqs. (19) and (20) and then adding them we get:

$$
\left(2 \epsilon \zeta A_{0}\right)^{2}+\left(\epsilon \alpha A_{0}+\frac{3}{4} \epsilon \beta A_{0}^{3}\right)^{2}=(\epsilon Y)^{2} .
$$

By solving Eq. (21) the amplitude of the response can be obtained. The parameter $\epsilon \alpha$ is not an independent parameter and hence needs to be eliminated. This can be done by defining $\omega_{0}^{2}=\Omega^{2}(1+\epsilon \alpha)$ where $\omega_{0}$ is the natural frequency of the associated linear system, that is with $\beta=0$. Using the above 
definition and re-arranging the terms, the zero order frequencyresponse function becomes:

$$
\begin{array}{r}
\left(2 \epsilon \zeta A_{0} \Omega^{2}\right)^{2}+\left(\omega_{0}^{2}\left[A_{0}+\frac{3}{4} \epsilon \beta A_{0}^{3}\right]-\Omega^{2} A_{0}\right)^{2}= \\
(\epsilon Y)^{2} \Omega^{4}
\end{array}
$$

The above equation can be expanded to form a polynomial equation as given below, and then can be solved for $A_{0}$.

$$
\begin{aligned}
& \frac{9}{16}(\epsilon \beta)^{2} \omega_{0}^{4} A_{0}^{6}+\frac{3}{2}(\epsilon \beta) \omega_{0}^{2}\left(\omega_{0}^{2}-\Omega^{2}\right) A_{0}^{4} \\
& +\left(\left(\omega_{0}^{2}-\Omega^{2}\right)^{2}+\left(2 \epsilon \zeta \Omega^{2}\right)^{2}\right) A_{0}^{2}-(\epsilon Y)^{2} \Omega^{4}=0
\end{aligned}
$$

Taking $S=A_{0}^{2}$, it becomes a cubic equation as:

$$
\begin{aligned}
& \frac{9}{16}(\epsilon \beta)^{2} \omega_{0}^{4} S^{3}+\frac{3}{2}(\epsilon \beta) \omega_{0}^{2}\left(\omega_{0}^{2}-\Omega^{2}\right) S^{2} \\
& \quad+\left(\left(\omega_{0}^{2}-\Omega^{2}\right)^{2}+\left(2 \epsilon \zeta \Omega^{2}\right)^{2}\right) S-(\epsilon Y)^{2} \Omega^{4}=0 .
\end{aligned}
$$

Equation (24) gives the zero order frequency-response function for the absolute displacement of a damped nonlinear system for a harmonic base excitation. The zero order solution is given by Eq. (16) and $A_{0}$ can be determined by solving Eq. (24).

The phase difference, which is the difference with respect to the phase of the excitation, can be shown to be:

$$
\tan \varphi_{0}=\frac{2 \epsilon \zeta}{\epsilon \alpha+\frac{3}{4} \epsilon \beta A_{0}^{2}}
$$

\section{RESPONSE FOR VARIOUS PARAMETERS OF THE SYSTEM}

The responses of the system for its various parameters are determined through solving Eq. (24) and the results are given in the following figures. The responses shown are the amplitudes of the zero order approximation $\left(A_{0}\right)$.

The response characteristics are shown in Figs. 2-6. The damping factor considered is 0.05 . In all these figures the amplitudes of the absolute displacement are plotted against the frequency of excitation for a system having a particular value of linearised natural frequency. The characteristics are given for five different linearised natural frequencies $\left(\omega_{0}\right) 200,400$, $600,800,1000 \mathrm{rad} / \mathrm{s}$. For a particular value of linearised natural frequency of the oscillator, the responses are shown for 6 values of amplitudes of base excitation. The amplitudes of excitation are $0.1 \mathrm{~mm}, 0.5 \mathrm{~mm}, 1 \mathrm{~mm}, 1.5 \mathrm{~mm}, 2 \mathrm{~mm}$ and $2.5 \mathrm{~mm}$. All the above curves are presented in one figure. All the curves in the same figure are drawn for a particular value of nonlinear spring parameter $\epsilon \beta$ in $\mathrm{N} / \mathrm{mm}^{2}$ and it is indicated in the title of the figure. The amplitudes of responses are given in millimeters.

Figures 2-7 provide the above characteristics for different values of nonlinear spring parameter $\epsilon \beta$. Figures $2-4$ give the responses respectively for $\epsilon \beta=0.001, \epsilon \beta=0.005$ and $\epsilon \beta=0.01$, when the spring is a hardening type. The results of systems with a softening type are shown in Figs. 5 and 6, Fig. 5 is for $\epsilon \beta=0.005$ and Fig. 6 is for $\epsilon \beta=0.01$.

The jump phenomenon in the response is seen as in forced excitation. The curves showing the variation of amplitude with the excitation frequency converge to the curve corresponding to $\epsilon Y=0$. In a linear system, they asymptotically approach $\Omega=\omega_{0}$, when the damping is absent. In a nonlinear system, that is $\epsilon \beta \neq 0$, they asymptotically approach a parabola, the equation of which can be derived as:

$$
A_{0}^{2}=\frac{4}{3 \epsilon \beta}\left\{\left(\frac{\Omega}{\omega_{0}}\right)^{2}-1\right\} .
$$

The asymptote intersects the $\Omega$-axis at $\Omega=\omega_{0}$.

It is important to note that the responses estimated using the expression derived in this work show very low values of responses when the frequency of excitation is low. The amplitude of response should have been approximately equal to the amplitude of excitation. The amplitude of response estimated is the zero order approximation. This issue is resolved by incorporating the first order approximation terms. This is presented subsequently.

\section{FIRST ORDER APPROXIMATION OF RESPONSE}

The issue seen in the results at lower frequencies is investigated here.

As discussed previously the displacement response can be expanded as per Eq. (7) and the phase of the response can be expanded as per Eq. (8). The zero order approximation to the solution is $x_{0}=A_{0} \cos \tau$. Solving the differential equation

$$
x_{1}^{\prime \prime}+x_{1}=-\frac{1}{4} \beta A_{0}^{3} \cos 3 \tau ;
$$

the first order approximation to the solution can be obtained as:

$$
x_{1}=A_{1} \cos \tau+\frac{1}{32} \beta A_{0}^{3} \cos 3 \tau .
$$

Combining both, the solution becomes:

$$
x(\tau)=\left(A_{0}+\epsilon A_{1}\right) \cos \tau+\frac{1}{32} \epsilon \beta A_{0}^{3} \cos 3 \tau+\ldots .
$$

The value of $A_{0}$ can be determined using Eq. (24). The response estimated using $A_{0}$ alone, that is without $A_{1}$, is found to be not correct in the low frequencies. Therefore, $A_{1}$ is determined and investigated whether incorporation of it improves the results.

\subsection{Expression for $A_{1}$}

The differential equation of motion is:

$$
\begin{aligned}
& \Omega^{2} x^{\prime \prime}+\omega^{2} x=\epsilon\left[-\omega^{2}\left(\alpha x+\beta x^{3}\right)-2 \zeta \omega \Omega x^{\prime}\right] \\
& -2 \epsilon \zeta \omega \Omega \epsilon Y \sin (\tau+\varphi)+\omega^{2} \epsilon Y \cos (\tau+\varphi) \\
& +\epsilon \omega^{2}\left[\epsilon \alpha Y \cos (\tau+\varphi)+\beta \epsilon^{3} Y^{3} \cos ^{3}(\tau+\varphi)\right] .
\end{aligned}
$$




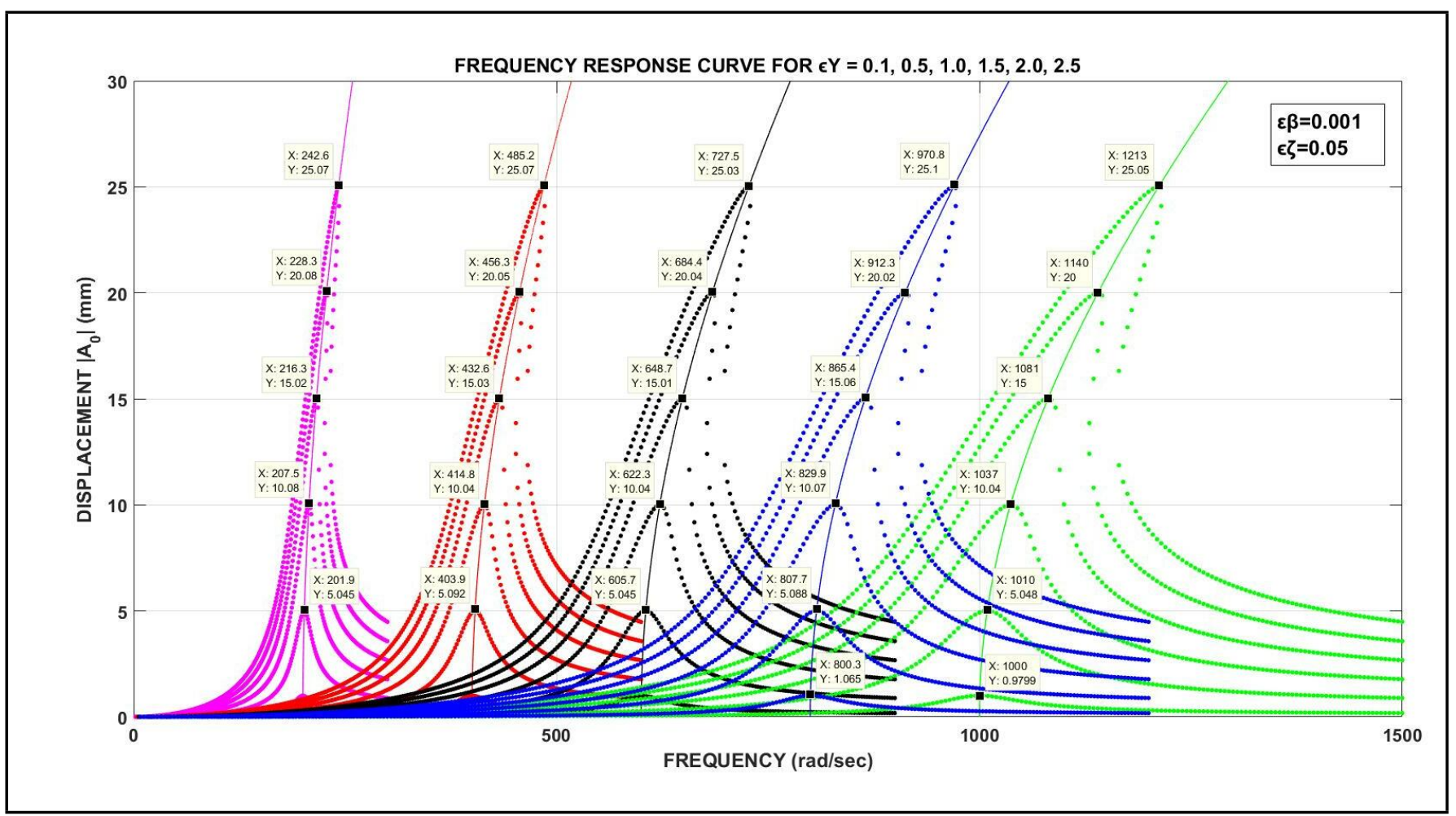

Figure 2. Frequency response of hard spring $(\epsilon \beta=0.001)$ for various values of excitations.

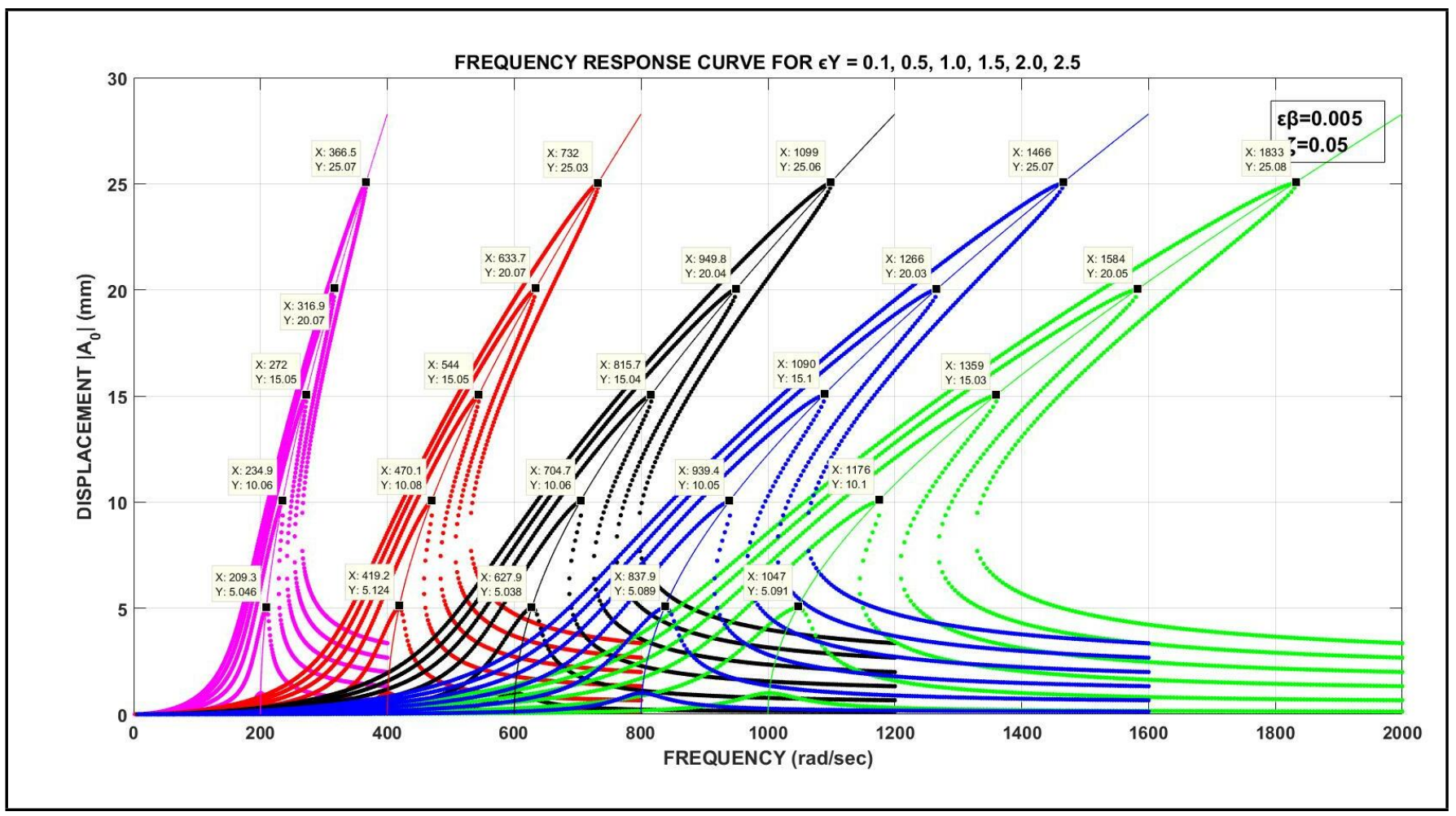

Figure 3. Frequency response of hard spring $(\epsilon \beta=0.005)$ for various values of excitations. 


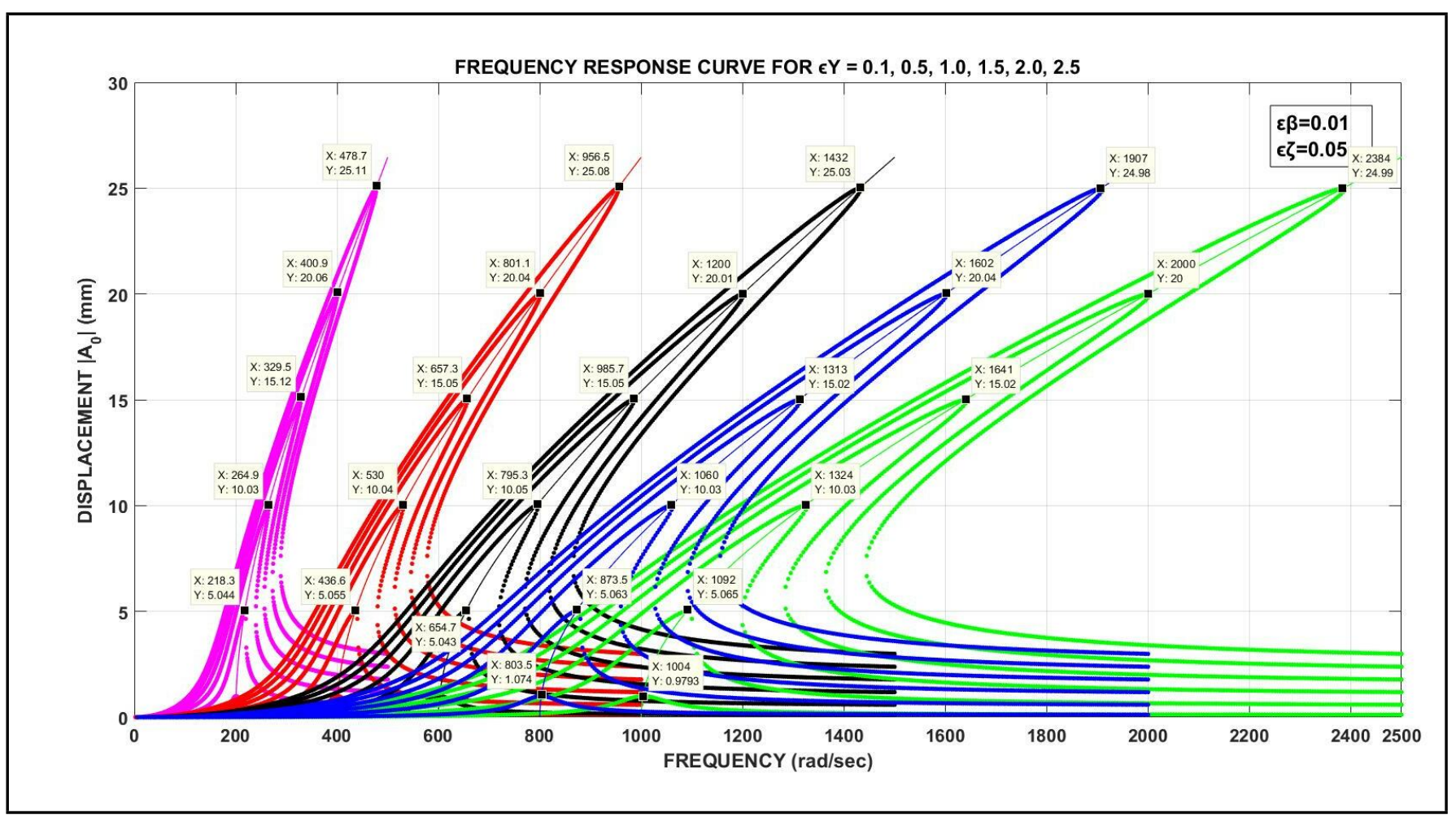

Figure 4. Frequency response of hard spring $(\epsilon \beta=0.01)$ for various values of excitations.

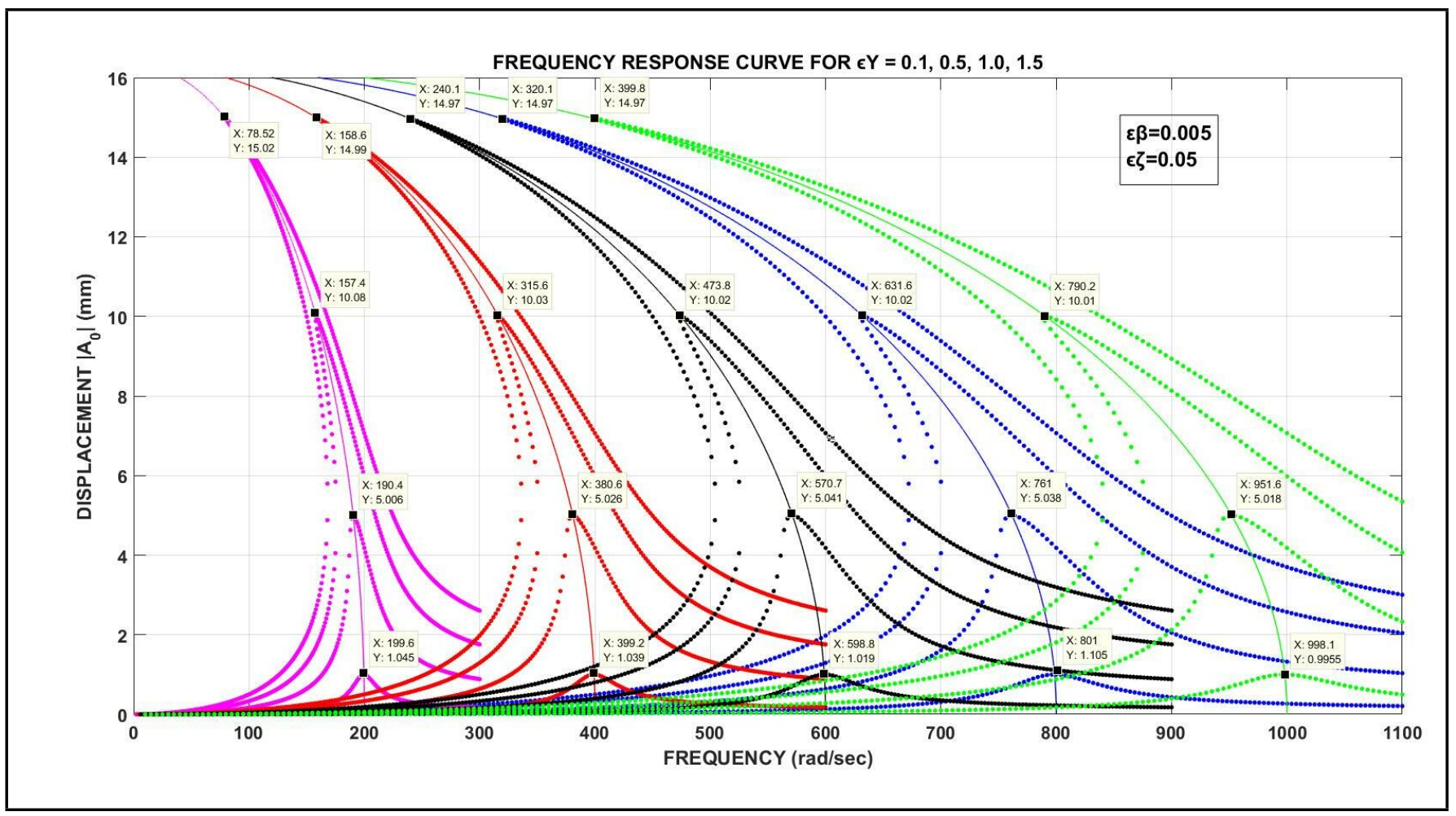

Figure 5. Frequency response of soft spring $(\epsilon \beta=0.005)$ for various values of excitations. 


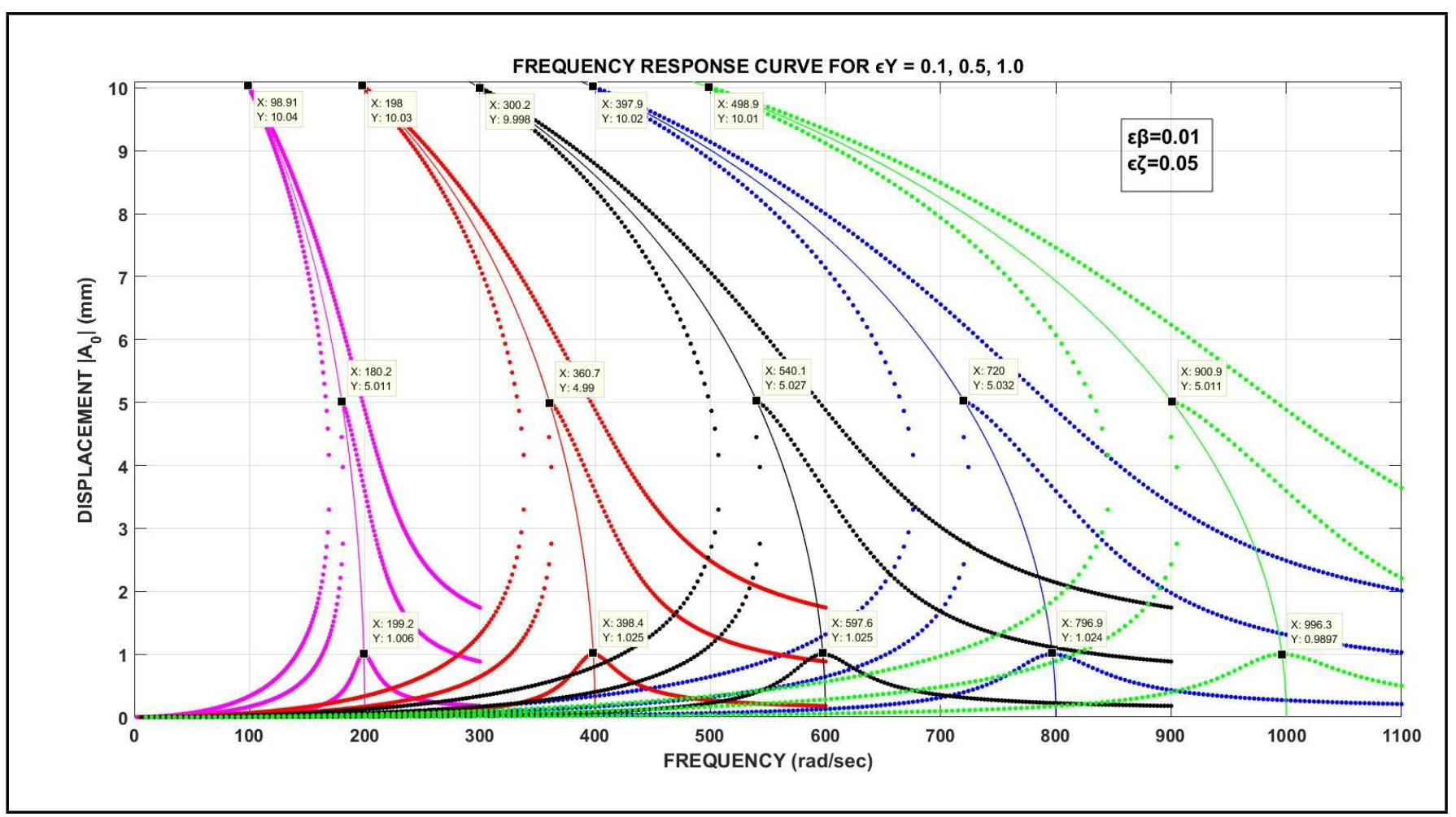

Figure 6. Frequency response of soft spring $(\epsilon \beta=0.01)$ for various values of excitations.

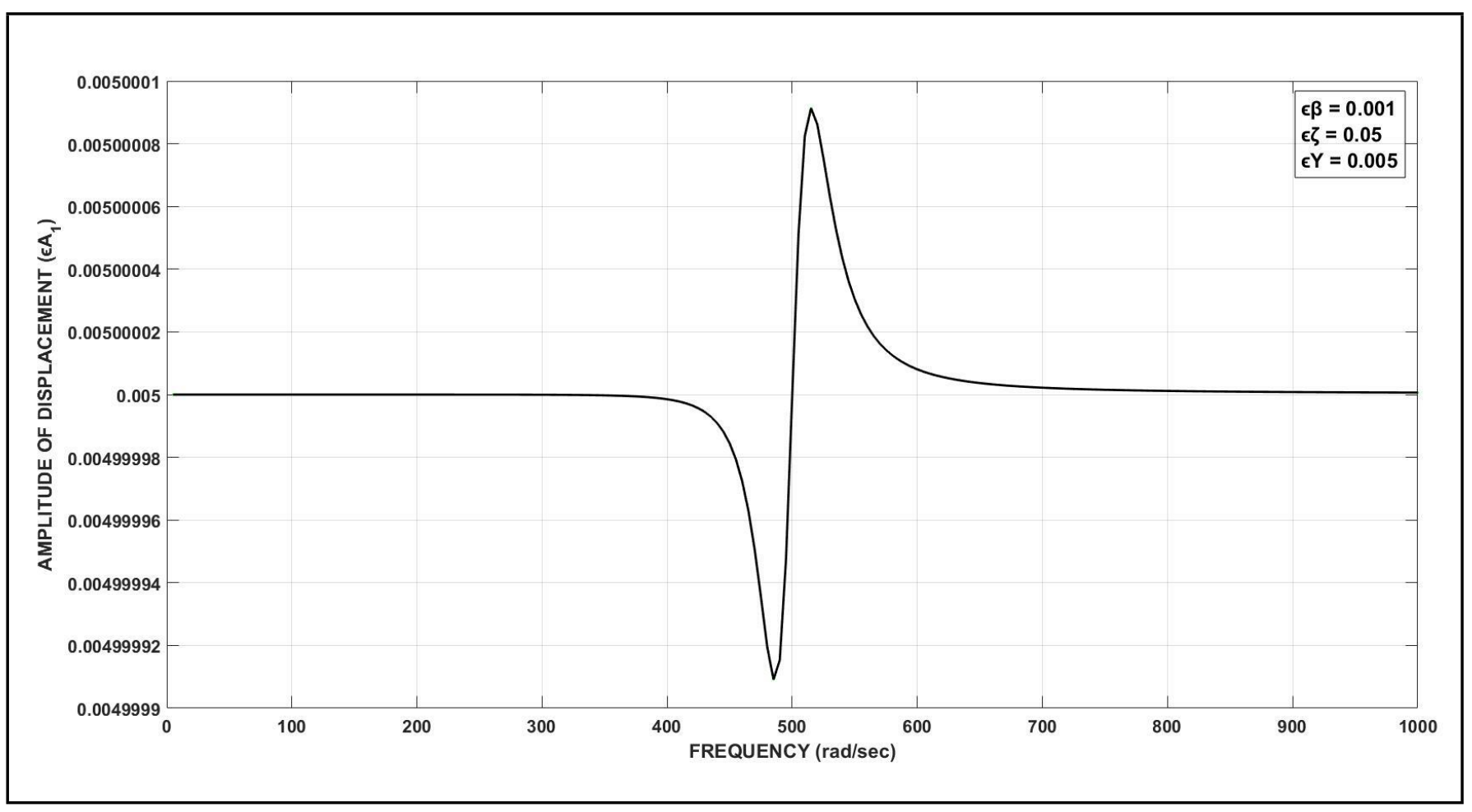

Figure 7. Frequency response $\left(\epsilon A_{1}\right)$ of hard spring $(\epsilon \beta=0.001)$. 
Using the solution for $x$ in terms of $x_{1}, x_{2}$ etc., the differential equation becomes:

$$
\begin{gathered}
\Omega^{2}\left(x_{0}+\epsilon x_{1}+\epsilon^{2} x_{2}\right)^{\prime \prime}+\omega^{2}\left(x_{0}+\epsilon x_{1}+\epsilon^{2} x_{2}\right)= \\
\epsilon\left[-\omega^{2}\left(\alpha\left[x_{0}+\epsilon x_{1}+\epsilon^{2} x_{2}\right]+\beta\left[x_{0}+\epsilon x_{1}+\epsilon^{2} x_{2}\right]^{3}\right)\right. \\
\left.-2 \zeta \omega \Omega\left(x_{0}+\epsilon x_{1}+\epsilon^{2} x_{2}\right)^{\prime}+\omega^{2} Y \cos (\tau+\varphi)\right] \\
+\epsilon^{2}\left(-2 \zeta \omega \Omega Y \sin (\tau+\varphi)+\omega^{2} \alpha Y \cos (\tau+\varphi)\right) \\
+\epsilon^{4} \omega^{2} \beta Y^{3} \cos ^{3}(\tau+\varphi)
\end{gathered}
$$

For small values of $\epsilon$, the values of higher powers of $\epsilon$ are negligible and hence:

$$
\left[x_{0}+\epsilon x_{1}+\epsilon^{2} x_{2}\right]^{3}=\left(x_{0}^{3}+\epsilon 3 x_{0}^{2} x_{1}\right) .
$$

Using Eq. (32) the differential equation becomes:

$$
\begin{gathered}
\Omega^{2}\left(x_{0}+\epsilon x_{1}+\epsilon^{2} x_{2}\right)^{\prime \prime}+\omega^{2}\left(x_{0}+\epsilon x_{1}+\epsilon^{2} x_{2}\right)= \\
\epsilon\left[-\omega^{2}\left(\alpha\left[x_{0}+\epsilon x_{1}+\epsilon^{2} x_{2}\right]+\beta\left(x_{0}^{3}+\epsilon 3 x_{0}{ }^{2} x_{1}\right)\right)\right. \\
\left.-2 \zeta \omega \Omega\left(x_{0}+\epsilon x_{1}+\epsilon^{2} x_{2}\right)^{\prime}+\omega^{2} Y \cos (\tau+\varphi)\right] \\
+\epsilon^{2}\left(-2 \zeta \omega \Omega Y \sin (\tau+\varphi)+\omega^{2} \alpha Y \cos (\tau+\varphi)\right) \\
+\epsilon^{4} \omega^{2} \beta Y^{3} \cos ^{3}(\tau+\varphi) .
\end{gathered}
$$

Expanding the differentials, we get:

$$
\begin{gathered}
\left(\Omega^{2} x_{0}^{\prime \prime}+\omega^{2} x_{0}\right)+\epsilon\left(\Omega^{2} x_{1}^{\prime \prime}+\omega^{2} x_{1}\right) \\
+\epsilon^{2}\left(\Omega^{2} x_{2}^{\prime \prime}+\omega^{2} x_{2}\right)= \\
\epsilon\left[-\omega^{2}\left(\alpha x_{0}+\beta x_{0}^{3}\right)-2 \zeta \omega \Omega x_{0}^{\prime}+\omega^{2} Y \cos (\tau+\varphi)\right] \\
\quad+\epsilon^{2}\left[-\omega^{2}\left(\alpha x_{1}+3 \beta x_{0}^{2} x_{1}\right)-2 \zeta \omega \Omega x_{1}^{\prime}\right. \\
\left.-2 \zeta \omega \Omega Y \sin (\tau+\varphi)+\omega^{2} \alpha Y \cos (\tau+\varphi)\right]
\end{gathered}
$$

Expanding $\varphi$ as given by Eq. (8) and using the trigonometric expansions we get:

$$
\begin{aligned}
\cos \left(\tau+\varphi_{0}+\epsilon \varphi_{1}+\epsilon^{2} \varphi_{2}\right) & = \\
\cos \left(\tau+\varphi_{0}\right)-\epsilon \varphi_{1} \sin ( & \left.\tau+\varphi_{0}\right)-\epsilon^{2} \varphi_{2} \sin \left(\tau+\varphi_{0}\right) \\
& -\epsilon \varphi_{1} * \epsilon^{2} \varphi_{2} \cos \left(\tau+\varphi_{0}\right)
\end{aligned}
$$

$$
\begin{aligned}
\sin \left(\tau+\varphi_{0}+\epsilon \varphi_{1}+\epsilon^{2} \varphi_{2}\right) & = \\
\sin \left(\tau+\varphi_{0}\right)+\epsilon \varphi_{1} \cos ( & \left.+\varphi_{0}\right)+\epsilon^{2} \varphi_{2} \cos \left(\tau+\varphi_{0}\right) \\
& -\epsilon \varphi_{1} * \epsilon^{2} \varphi_{2} \sin \left(\tau+\varphi_{0}\right)
\end{aligned}
$$

by approximating for the sin and cos of small angles. As value of $\epsilon$ is very small, the values of $\epsilon^{2}$ and $\epsilon^{3}$ can be neglected. Therefore, one can approximate the cosine and sine expansions in Eq. (35) and (36) as:

$$
\cos \left(\tau+\varphi_{0}+\epsilon \varphi_{1}+\epsilon^{2} \varphi_{2}\right)=\cos \left(\tau+\varphi_{0}\right)-\epsilon \varphi_{1} \sin \left(\tau+\varphi_{0}\right)
$$

$\sin \left(\tau+\varphi_{0}+\epsilon \varphi_{1}+\epsilon^{2} \varphi_{2}\right)=\sin \left(\tau+\varphi_{0}\right)+\epsilon \varphi_{1} \cos \left(\tau+\varphi_{0}\right)$.
Applying Eq. (37) and (38) the differential equation becomes:

$$
\begin{gathered}
\left(\Omega^{2} x_{0}^{\prime \prime}+\omega^{2} x_{0}\right)+\epsilon\left(\Omega^{2} x_{1}^{\prime \prime}+\omega^{2} x_{1}\right) \\
+\epsilon^{2}\left(\Omega^{2} x_{2}^{\prime \prime}+\omega^{2} x_{2}\right)= \\
\epsilon\left[-\omega^{2}\left(\alpha x_{0}+\beta x_{0}^{3}\right)-2 \zeta \omega \Omega x_{0}^{\prime}\right. \\
\left.+\omega^{2} Y\left\{\cos \left(\tau+\varphi_{0}\right)-\epsilon \varphi_{1} \sin \left(\tau+\varphi_{0}\right)-\epsilon^{2} \varphi_{2} \sin \left(\tau+\varphi_{0}\right)\right\}\right] \\
+\epsilon^{2}\left[-\omega^{2}\left(\alpha x_{1}+3 \beta x_{0}^{2} x_{1}\right)-2 \zeta \omega \Omega x_{1}^{\prime}\right. \\
-2 \zeta \omega \Omega Y\left\{\sin \left(\tau+\varphi_{0}\right)+\epsilon \varphi_{1} \cos \left(\tau+\varphi_{0}\right)\right. \\
\left.+\epsilon^{2} \varphi_{2} \cos \left(\tau+\varphi_{0}\right)\right\}+\omega^{2} \alpha Y\left\{\cos \left(\tau+\varphi_{0}\right)\right. \\
\left.\left.-\epsilon \varphi_{1} \sin \left(\tau+\varphi_{0}\right)-\epsilon^{2} \varphi_{2} \sin \left(\tau+\varphi_{0}\right)\right\}\right] .
\end{gathered}
$$

On neglecting higher powers of $\epsilon$ :

$$
\begin{gathered}
\left(\Omega^{2} x_{0}^{\prime \prime}+\omega^{2} x_{0}\right)+\epsilon\left(\Omega^{2} x_{1}^{\prime \prime}+\omega^{2} x_{1}\right) \\
+\epsilon^{2}\left(\Omega^{2} x_{2}^{\prime \prime}+\omega^{2} x_{2}\right)= \\
\epsilon\left[-\omega^{2}\left(\alpha x_{0}+\beta x_{0}^{3}\right)-2 \zeta \omega \Omega x_{0}^{\prime}+\omega^{2} Y \cos \left(\tau+\varphi_{0}\right)\right] \\
+\epsilon^{2}\left[-\omega^{2}\left(\alpha x_{1}+3 \beta x_{0}^{2} x_{1}\right)-2 \zeta \omega \Omega x_{1}^{\prime}\right. \\
-\omega^{2} Y \varphi_{1} \sin \left(\tau+\varphi_{0}\right)-2 \zeta \omega \Omega Y \sin \left(\tau+\varphi_{0}\right) \\
\left.+\omega^{2} \alpha Y \cos \left(\tau+\varphi_{0}\right)\right] .
\end{gathered}
$$

Equating the coefficients of $\epsilon^{0}$, the zero order approximation of the differential equation becomes:

$$
\Omega^{2} x_{0}^{\prime \prime}+\omega^{2} x_{0}=0
$$

Equating the coefficients of $\epsilon^{1}$ we get:

$$
\begin{aligned}
& \Omega^{2} x_{1}^{\prime \prime}+\omega^{2} x_{1}= \\
& -\omega^{2}\left(\alpha x_{0}+\beta x_{0}^{3}\right)-2 \zeta \omega \Omega x_{0}^{\prime}+\omega^{2} Y \cos \left(\tau+\varphi_{0}\right) .
\end{aligned}
$$

Equating the coefficients of $\epsilon^{2}$, the differential equation becomes:

$$
\begin{aligned}
& \Omega^{2} x_{2}^{\prime \prime}+\omega^{2} x_{2}=-\omega^{2}\left(\alpha x_{1}+3 \beta x_{0}^{2} x_{1}\right) \\
&-2 \zeta \omega \Omega x_{1}^{\prime}-\omega^{2} Y \varphi_{1} \sin \left(\tau+\varphi_{0}\right)-2 \zeta \omega \Omega Y \\
& \sin \left(\tau+\varphi_{0}\right)+\omega^{2} \alpha Y \cos \left(\tau+\varphi_{0}\right)
\end{aligned}
$$

The zero order approximation to the solution is as given by Eq. (16) and the first order approximation is as given by Eq. (28). Second order approximation can be obtained from Eq. (43), which can be written as:

$$
\begin{aligned}
& \Omega^{2} x_{2}^{\prime \prime}+\omega^{2} x_{2}=-\omega^{2}\left(\alpha x_{1}+3 \beta x_{0}^{2} x_{1}\right)-2 \zeta \omega \Omega x_{1}^{\prime} \\
& -\left\{\omega^{2} Y \varphi_{1}+2 \zeta \omega \Omega Y\right\}\left[\cos (\tau) * \sin \left(\varphi_{0}\right)+\cos \left(\varphi_{0}\right) * \sin (\tau)\right] \\
& \quad+\omega^{2} \alpha Y\left[\cos (\tau) * \cos \left(\varphi_{0}\right)-\sin (\tau) * \sin \left(\varphi_{0}\right)\right] .
\end{aligned}
$$

Using the solutions for $x_{0}$ and $x_{1}$ and dividing Eq. (44) by 


\section{$\omega^{2}$ we get:}

$$
\begin{aligned}
& x_{2}^{\prime \prime}+x_{2}=-\left(\alpha\left\{A_{1} \cos \tau+\frac{1}{32} \beta A_{0}^{3} \cos 3 \tau\right\}\right. \\
&\left.+3 \beta\left\{A_{0} \cos \tau\right\}^{2}\left\{A_{1} \cos \tau+\frac{1}{32} \beta A_{0}^{3} \cos 3 \tau\right\}\right) \\
&-2 \zeta\left\{-A_{1} \sin \tau-\frac{3}{32} \beta A_{0}^{3} \sin 3 \tau\right\} \\
&-\left\{Y \varphi_{1}+2 \zeta Y\right\}\left(\cos \tau \sin \varphi_{0}+\cos \varphi_{0} \sin \tau\right) \\
&+\alpha Y\left(\cos \tau \cos \varphi_{0}-\sin \tau \sin \varphi_{0}\right) .
\end{aligned}
$$

Re-arranging the terms we get:

$$
\begin{gathered}
x_{2}^{\prime \prime}+x_{2}=-\left(\alpha A_{1} \cos \tau+\frac{1}{32} \alpha \beta A_{0}^{3} \cos 3 \tau+\right. \\
+3 \beta A_{0}^{2} A_{1} \cos ^{3} \tau \\
\left.+\frac{3}{32} \beta^{2} A_{0}^{5} \cos 3 \tau \cos ^{2} \tau\right)-\left(-2 \zeta A_{1} \sin \tau-\frac{3}{16} \zeta \beta A_{0}^{3} \sin 3 \tau\right) \\
-\left\{Y \varphi_{1}+2 \zeta Y\right\}\left(\cos \tau \sin \varphi_{0}+\cos \varphi_{0} \sin \tau\right) \\
+\alpha Y\left(\cos \tau \cos \varphi_{0}-\sin \tau \sin \varphi_{0}\right) .
\end{gathered}
$$

Using the trigonometric relations $\cos 3 \tau \cos ^{2} \tau=\frac{1}{4}(\cos \tau+$ $2 \cos 3 \tau+\cos 5 \tau)$ and $\cos ^{3} \tau=\frac{1}{4}(3 \cos \tau+\cos 3 \tau)$ the differential equation changes to:

$$
\begin{gathered}
x_{2}^{\prime \prime}+x_{2}=-\left(\alpha A_{1} \cos \tau+\frac{1}{32} \alpha \beta A_{0}^{3} \cos 3 \tau+\right. \\
\beta A_{0}^{2} A_{1}\left(\frac{1}{4}(3 \cos \tau+\cos 3 \tau)\right)+ \\
\left.\frac{3}{32} \beta^{2} A_{0}^{5}\left(\frac{1}{4}(\cos \tau+2 \cos 3 \tau+\cos 5 \tau)\right)\right) \\
-\left(-2 \zeta A_{1} \sin \tau-\frac{3}{16} \zeta \beta A_{0}^{3} \sin 3 \tau\right) \\
-\left\{Y \varphi_{1}+2 \zeta Y\right\}\left(\cos \tau \sin \varphi_{0}+\cos \varphi_{0} \sin \tau\right) \\
+\alpha Y\left(\cos \tau \cos \varphi_{0}-\sin \tau \sin \varphi_{0}\right) .
\end{gathered}
$$

Collecting all $\cos \tau, \cos 3 \tau$ and neglecting $\cos 5 \tau$ terms, we get:

$$
\begin{gathered}
x_{2}^{\prime \prime}+x_{2}=\cos \tau\left\{-\alpha A_{1}-\frac{9}{4} \beta A_{0}^{2} A_{1}-\frac{3}{128} \beta^{2} A_{0}^{5}\right. \\
\left.-\left(Y \varphi_{1}+2 \zeta Y\right) \sin \varphi_{0}+\alpha Y \cos \varphi_{0}\right\} \\
+\sin \tau\left\{2 \zeta A_{1}-\left(Y \varphi_{1}+2 \zeta Y\right) \cos \varphi_{0}-\alpha Y \sin \varphi_{0}\right\} \\
+\cos 3 \tau\left\{-\frac{1}{32} \alpha \beta A_{0}^{3}-\frac{3}{4} \beta A_{0}^{2} A_{1}-\frac{6}{128} \beta^{2} A_{0}^{5}\right\} \\
+\frac{3}{16} \zeta \beta A_{0}^{3} \sin 3 \tau .
\end{gathered}
$$

To avoid the secular terms, equating the coefficients of $\cos \tau$ and $\sin \tau$ to zero:

$$
\begin{aligned}
-\alpha A_{1}- & \frac{9}{4} \beta A_{0}^{2} A_{1}-\frac{3}{128} \beta^{2} A_{0}^{5} \\
& -\left(Y \varphi_{1}+2 \zeta Y\right) \sin \varphi_{0}+\alpha Y \cos \varphi_{0}=0 \\
2 \zeta A_{1}- & \left(Y \varphi_{1}+2 \zeta Y\right) \cos \varphi_{0}-\alpha Y \sin \varphi_{0}=0
\end{aligned}
$$

The aim now is to solve for $A_{1}$. Re-arranging the terms and removing the dependence on the phase angle:

$$
\begin{aligned}
& \left(Y \varphi_{1}+2 \zeta Y\right)^{2}+(\alpha Y)^{2}= \\
& \quad\left(\alpha A_{1}+\frac{9}{4} \beta A_{0}^{2} A_{1}+\frac{3}{128} \beta^{2} A_{0}^{5}\right)^{2}+\left(2 \zeta A_{1}\right)^{2} .
\end{aligned}
$$

Introducing $\epsilon$ and considering $\epsilon \varphi_{1} \ll 2 \epsilon \zeta$, we get:

$$
\begin{aligned}
& (2 \epsilon \zeta \epsilon Y)^{2}+(\epsilon \alpha \epsilon Y)^{2}= \\
& \left(\epsilon \alpha \epsilon A_{1}+\frac{9}{4} \epsilon \beta A_{0}{ }^{2} \epsilon A_{1}+\frac{3}{128}(\epsilon \beta)^{2} A_{0}^{5}\right)^{2}+\left(2 \epsilon \zeta \epsilon A_{1}\right)^{2} .
\end{aligned}
$$

As $\frac{\omega_{0}^{2}-\Omega^{2}}{\Omega^{2}}=\epsilon \alpha$, Eq. (52) gets modified as:

$$
\begin{aligned}
(2 \epsilon \zeta \epsilon Y)^{2}+\left(\frac{\omega_{0}^{2}-\Omega^{2}}{\Omega^{2}} \epsilon Y\right)^{2}= & \\
\left(\frac{\omega_{0}^{2}-\Omega^{2}}{\Omega^{2}} \epsilon A_{1}+\frac{9}{4} \epsilon \beta A_{0}^{2} \epsilon A_{1}+\right. & \left.\frac{3}{128}(\epsilon \beta)^{2} A_{0}^{5}\right)^{2} \\
& +\left(2 \epsilon \zeta \epsilon A_{1}\right)^{2}
\end{aligned}
$$

Expanding the square of the expression and collecting the terms involving $\epsilon A_{1}$ and $\left(\epsilon A_{1}\right)^{2}$ we get:

$$
\begin{gathered}
(2 \epsilon \zeta \epsilon Y)^{2}+\left(\frac{\omega_{0}^{2}-\Omega^{2}}{\Omega^{2}} \epsilon Y\right)^{2}= \\
\left(\left[\frac{\omega_{0}^{2}-\Omega^{2}}{\Omega^{2}}\right]^{2}+\left[\frac{9}{4} \epsilon \beta A_{0}^{2}\right]^{2}\right. \\
\left.+2\left[\frac{\omega_{0}^{2}-\Omega^{2}}{\Omega^{2}}\right]\left[\frac{9}{4} \epsilon \beta A_{0}^{2}\right]+[2 \epsilon \zeta]^{2}\right)\left(\epsilon A_{1}\right)^{2} \\
+\left(\frac{27}{256}(\epsilon \beta)^{3} A_{0}^{7}+\frac{6}{128}\left[\frac{\omega_{0}^{2}-\Omega^{2}}{\Omega^{2}}\right](\epsilon \beta)^{2} A_{0}^{5}\right)\left(\epsilon A_{1}\right) \\
+\left(\frac{3}{128}(\epsilon \beta)^{2} A_{0}^{5}\right)^{2}
\end{gathered}
$$

Simplifying the coefficient of $\left(\epsilon A_{1}\right)^{2}$, and re-arranging the terms:

$$
\begin{aligned}
& \left(\left\{\left[\frac{\omega_{0}^{2}-\Omega^{2}}{\Omega^{2}}\right]+\left[\frac{9}{4} \epsilon \beta A_{0}^{2}\right]\right\}^{2}+[2 \epsilon \zeta]^{2}\right)\left(\epsilon A_{1}\right)^{2} \\
& +\left(\frac{27}{256}(\epsilon \beta)^{3} A_{0}^{7}+\frac{6}{128}\left[\frac{\omega_{0}^{2}-\Omega^{2}}{\Omega^{2}}\right](\epsilon \beta)^{2} A_{0}^{5}\right)\left(\epsilon A_{1}\right) \\
& +\left(\frac{3}{128}(\epsilon \beta)^{2} A_{0}^{5}\right)^{2}-(2 \epsilon \zeta \epsilon Y)^{2}-\left(\frac{\omega_{0}^{2}-\Omega^{2}}{\Omega^{2}} \epsilon Y\right)^{2}=0 .
\end{aligned}
$$

The above relation is a quadratic equation in $\epsilon A_{1}$ as given be- 
low:

$$
\begin{gathered}
\left(\left\{\left[\frac{\omega_{0}^{2}-\Omega^{2}}{\Omega^{2}}\right]+\left[\frac{9}{4} \epsilon \beta A_{0}^{2}\right]\right\}^{2}+[2 \epsilon \zeta]^{2}\right)\left(\epsilon A_{1}\right)^{2} \\
+\frac{3}{128}(\epsilon \beta)^{2}\left(\frac{9}{2} \epsilon \beta A_{0}^{2}+2\left[\frac{\omega_{0}^{2}-\Omega^{2}}{\Omega^{2}}\right]\right) A_{0}^{5}\left(\epsilon A_{1}\right) \\
+\left(\frac{3}{128}(\epsilon \beta)^{2} A_{0}^{5}\right)^{2}-(2 \epsilon \zeta \epsilon Y)^{2}-\left(\frac{\omega_{0}^{2}-\Omega^{2}}{\Omega^{2}} \epsilon Y\right)^{2}=0 .
\end{gathered}
$$

Solving the above quadratic equation, $\epsilon A_{1}$ can be determined. The amplitude of the response in the fundamental frequency is $A_{0}+\epsilon A_{1}$.

\subsection{Results of $\epsilon A_{1}$}

The values of $\epsilon A_{1}$ for various values of nonlinear spring parameter, damping and excitation etc., are shown in Figs. 7-10 for hard and soft springs with linearised natural frequency of $500 \mathrm{rad} / \mathrm{s}$. The results show that the amplitude of response at low frequency is approximately the same as the amplitude of excitation. The problem in the low frequency can be resolved by incorporating the first order term.

\section{RESPONSE WITH ZERO AND FIRST ORDER APPROXIMATIONS}

It was discussed previously that the responses estimated using the expression derived in this work show very low value of response when the frequency of excitation is low. The amplitude of response should have been approximately equal to the amplitude of excitation. The amplitude of response estimated was with the zero order approximation and the first order approximation term was not included.

Here the amplitude of the response in the fundamental frequency of oscillation considering both zero order as well as the first order approximations $\left(A_{0}+\epsilon A_{1}\right)$, is plotted at various excitation frequencies and various parameters of the system. They are shown in Figs. 11-18 and are presented in the same way as before.

Figure 11 gives the responses for the nonlinear spring parameter $\epsilon \beta=0.001$, Fig. 12 gives the results for $\epsilon \beta=0.005$, Fig. 13 gives the results for $\epsilon \beta=0.01$ and Fig. 14 shows the results for $\epsilon \beta=0.05$ when the spring is hardening type. Figures 15-18 show similar result for a system with softening spring.

It can be seen that the problems encountered at low frequencies are resolved by incorporating the first order approximation term and the responses are on expected lines. The response near resonance is contributed by zero order term and the response at low frequencies are from the first order term. The values of $A_{0}$ can be determined by solving Eq. (24) and $\epsilon A_{1}$ can be determined by solving Eq. (56).

Following points can be noted from all the above response curves

- As the frequency of excitation is increased or decreased, a jump is seen in the magnitude of the response. As the excitation frequency is being increased the jump occurs at a higher frequency and when the excitation frequency is being decreased the jump occurs at a lower frequency.

- In the case of a hard spring with the increase in the magnitude of excitation $(\epsilon Y)$ the frequency at which the response is the maximum increases (for constant $\epsilon \beta$ and $\epsilon \zeta$ ). For the soft spring, the characteristics are reversed.

- In the case of a hard spring, the frequency at which the response is the maximum increases considerably with the increase in the non-linearity $(\epsilon \beta)$.

- For small values of nonlinearities, the amplification remains approximately the same for different values of excitations. Consequently, the maximum response will be proportional to the excitation and will be independent of nonlinear spring parameters. The behavior of a soft spring is identical to that of a hard spring with reverse characteristics.

- In the absence of damping, the response asymptotically converges to a parabola. This parabola intersects the $\Omega$ axis at $\Omega=\omega_{0}$.

- The response at the fundamental frequency leads the excitation by 90 degrees. This is considering the zero order approximation. The phase difference is zero if damping is absent, as in linear systems.

\section{CONCLUSIONS}

Responses of a system with nonlinear stiffness subjected to base harmonic excitation are determined using Lindstedt's method. An expression to estimate the amplitude of the fundamental frequency of oscillation is derived. It exhibits the jump phenomena as shown for forced excitations. The amplitudes of responses at various values of excitation frequencies are obtained for various nonlinear parameters of the oscillator. The existing expression for the amplitude of response for base excitation is derived from relative displacement making certain assumptions on phase. But the present expression is derived from first principles without these assumptions. Additionally, the expression for the phase difference and the expression for the asymptote where the responses converge are also derived.

The responses estimated using the expression derived in this work show very low value of response when the frequency of excitation is low. At low frequencies, amplitude of response should have been approximately equal to the amplitude of excitation. The amplitude of response estimated is the zero order approximation and the first order approximation was not included. Therefore, an expression for the first order approximation is derived. Its characteristics for various parameters of the system are obtained. By incorporating the first order term, the problem in the low frequency is resolved. Variation of the amplitude, incorporating both zero order term as well as the first order term, with the excitation frequency are presented for various nonlinear spring parameters of the oscillator. 


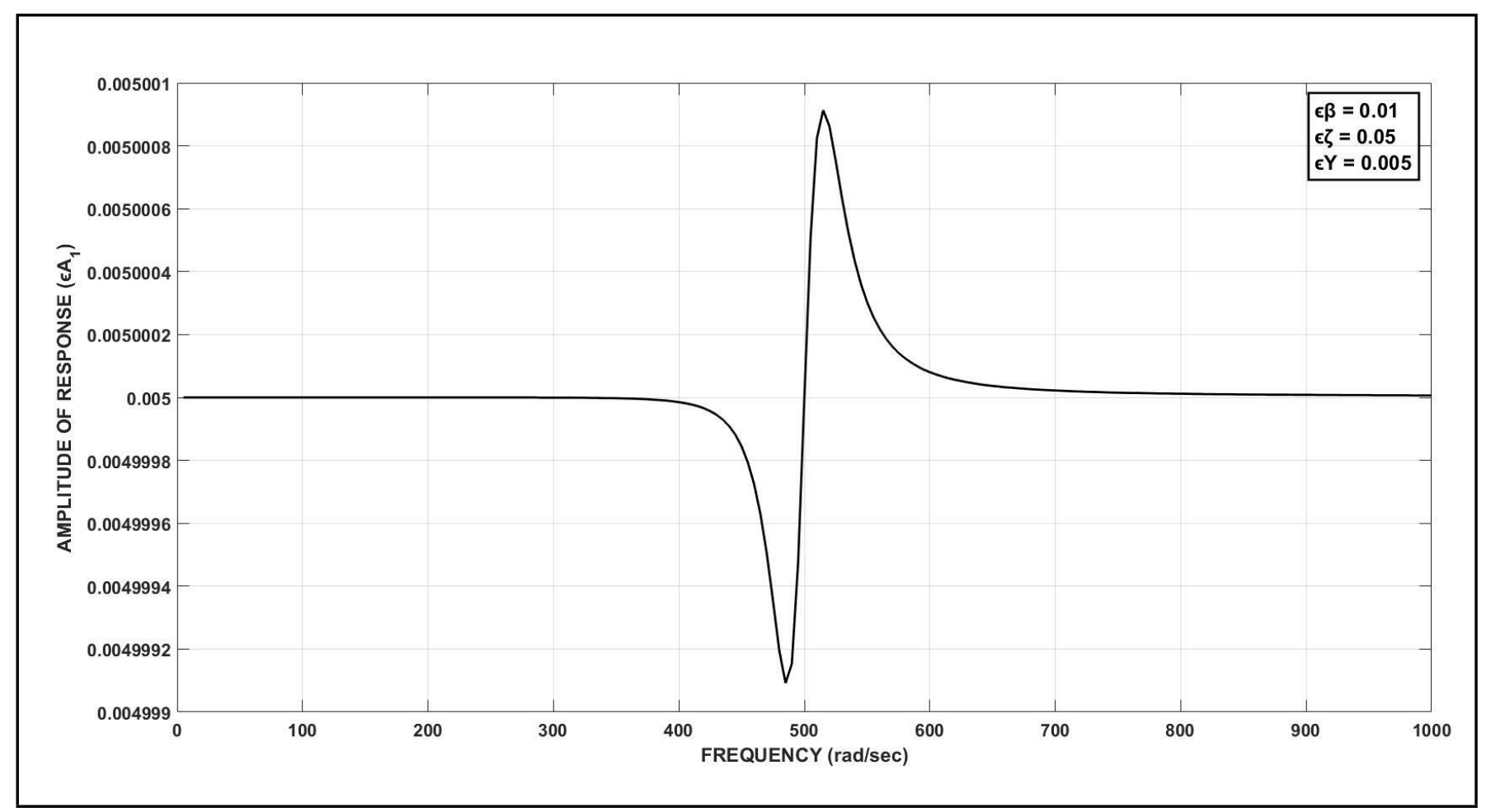

Figure 8. Frequency response $\left(\epsilon A_{1}\right)$ of hard spring $(\epsilon \beta=0.01)$.

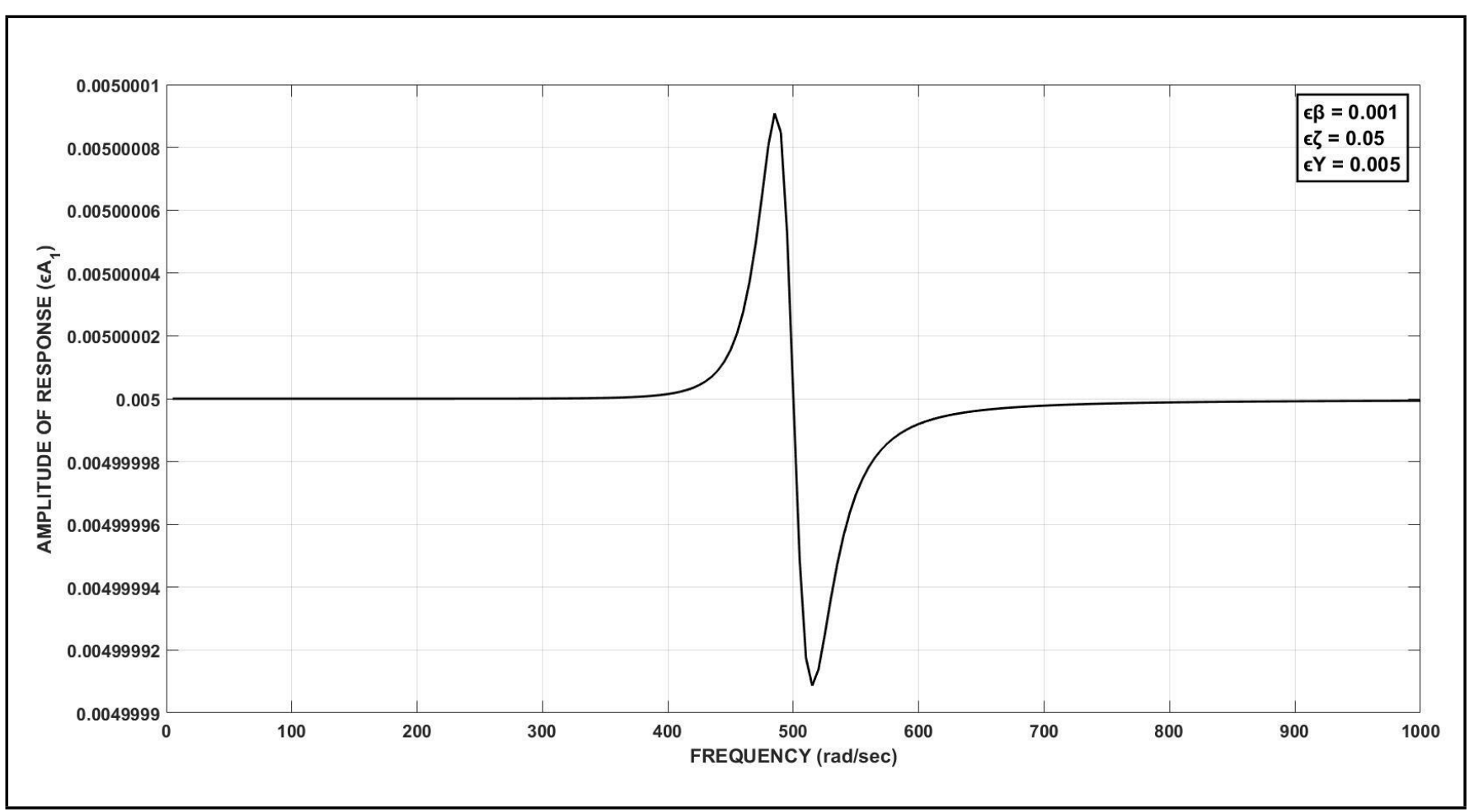

Figure 9. Frequency response $\left(\epsilon A_{1}\right)$ of soft spring $(\epsilon \beta=0.001)$. 


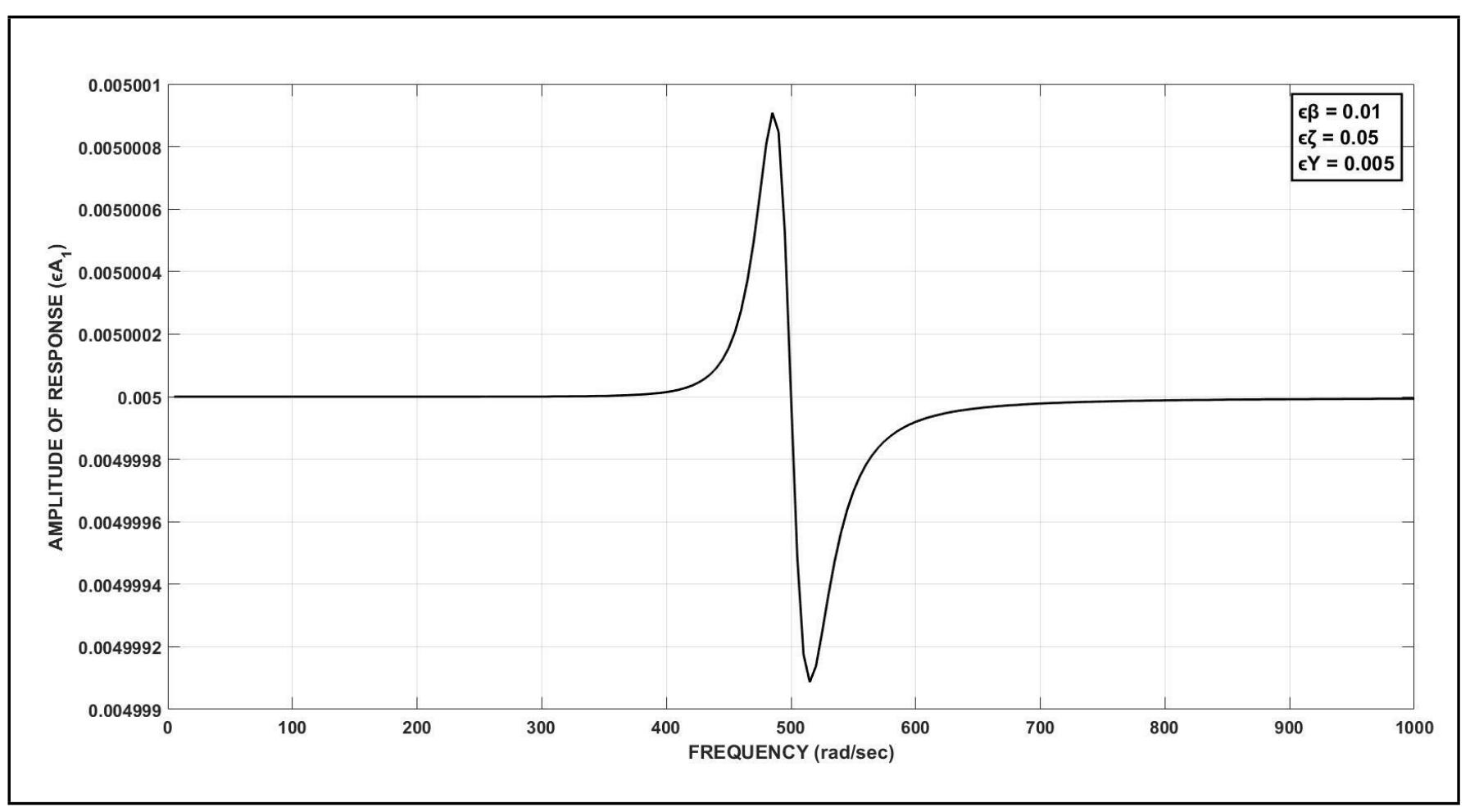

Figure 10. Frequency response $\left(\epsilon A_{1}\right)$ of soft spring $(\epsilon \beta=0.01)$.

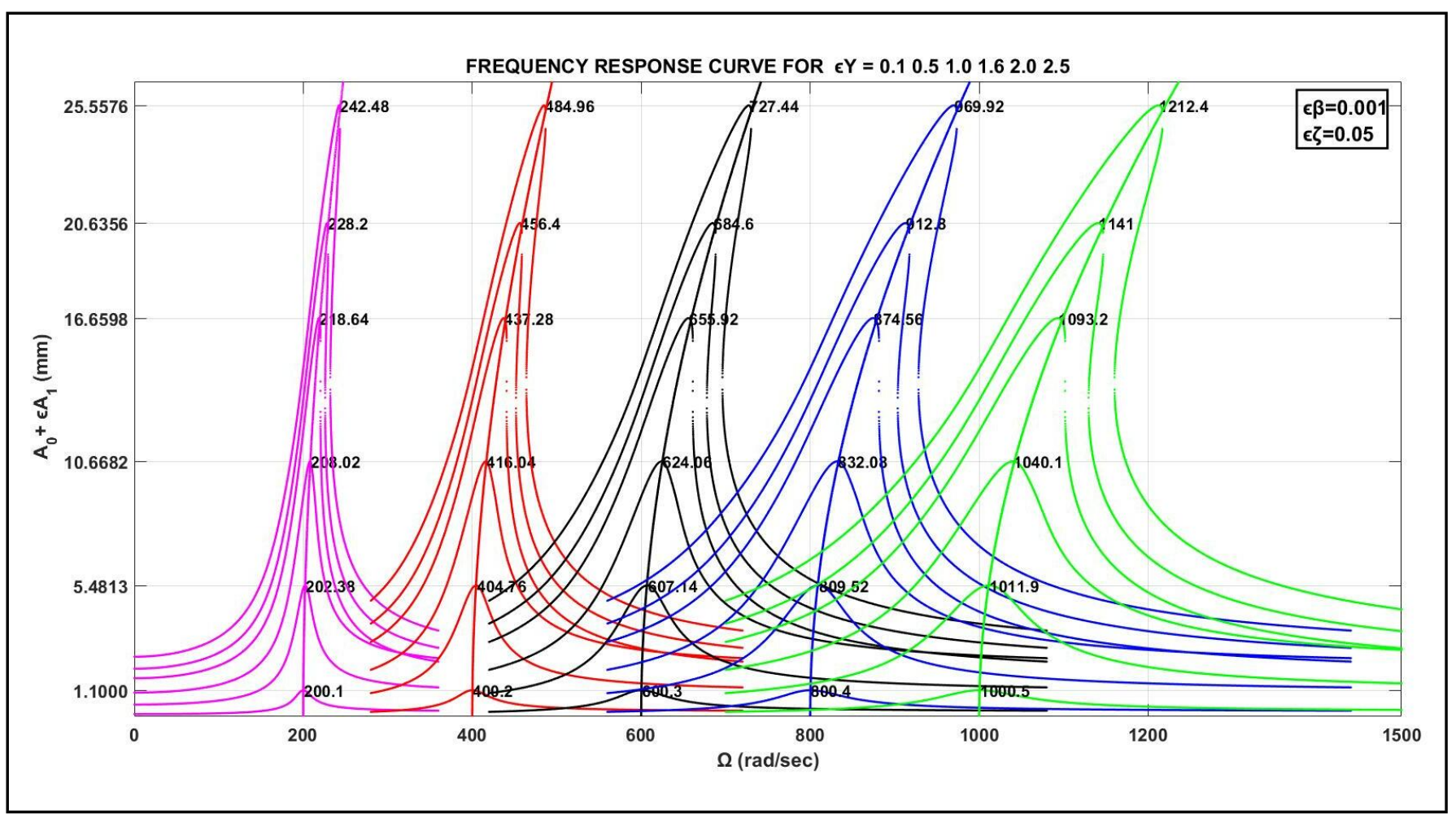

Figure 11. Frequency response $\left(A_{0}+\epsilon A_{1}\right)$ of hard spring $(\epsilon \beta=0.001)$. 


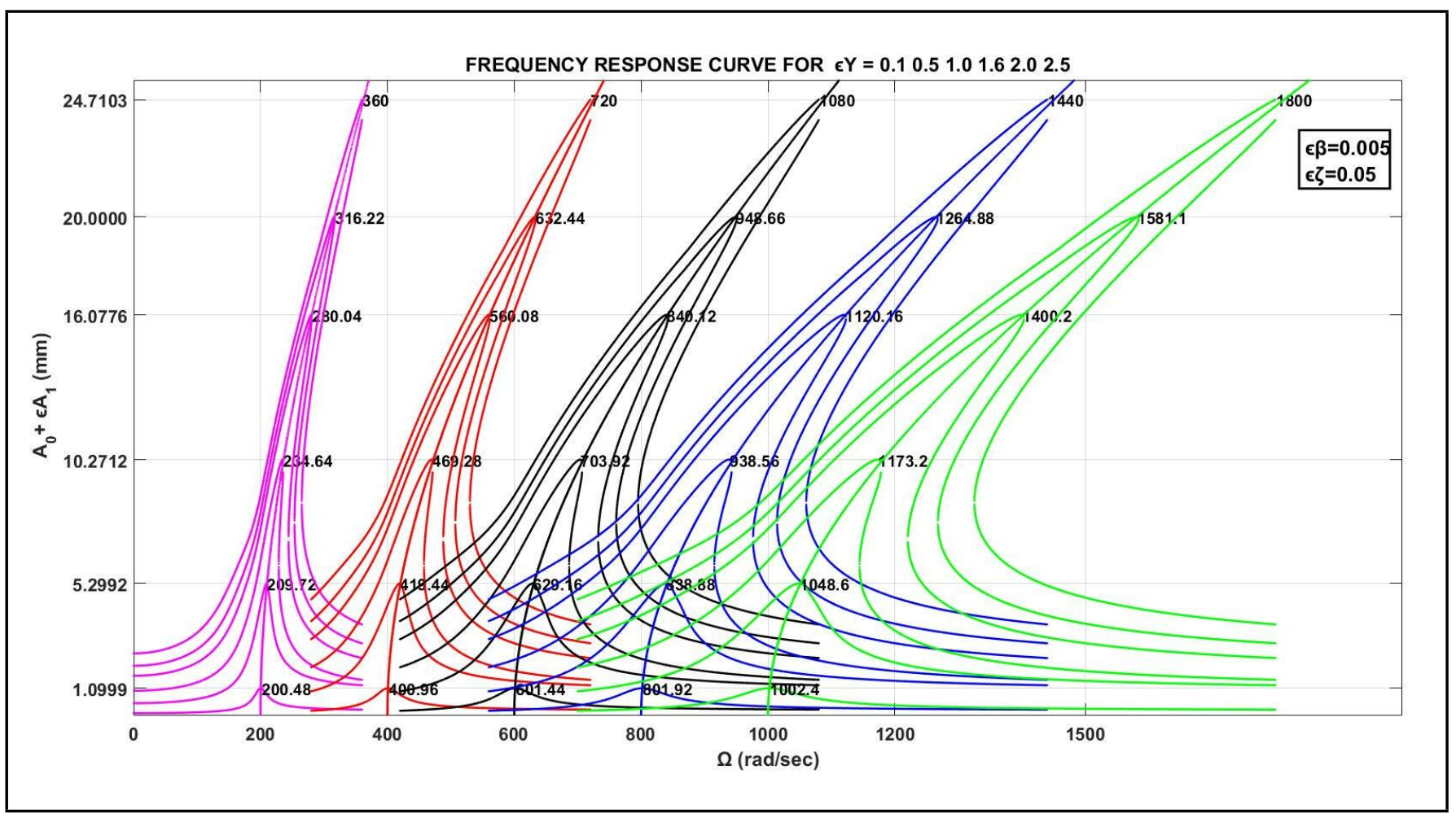

Figure 12. Frequency response $\left(A_{0}+\epsilon A_{1}\right)$ of hard spring $(\epsilon \beta=0.005)$.

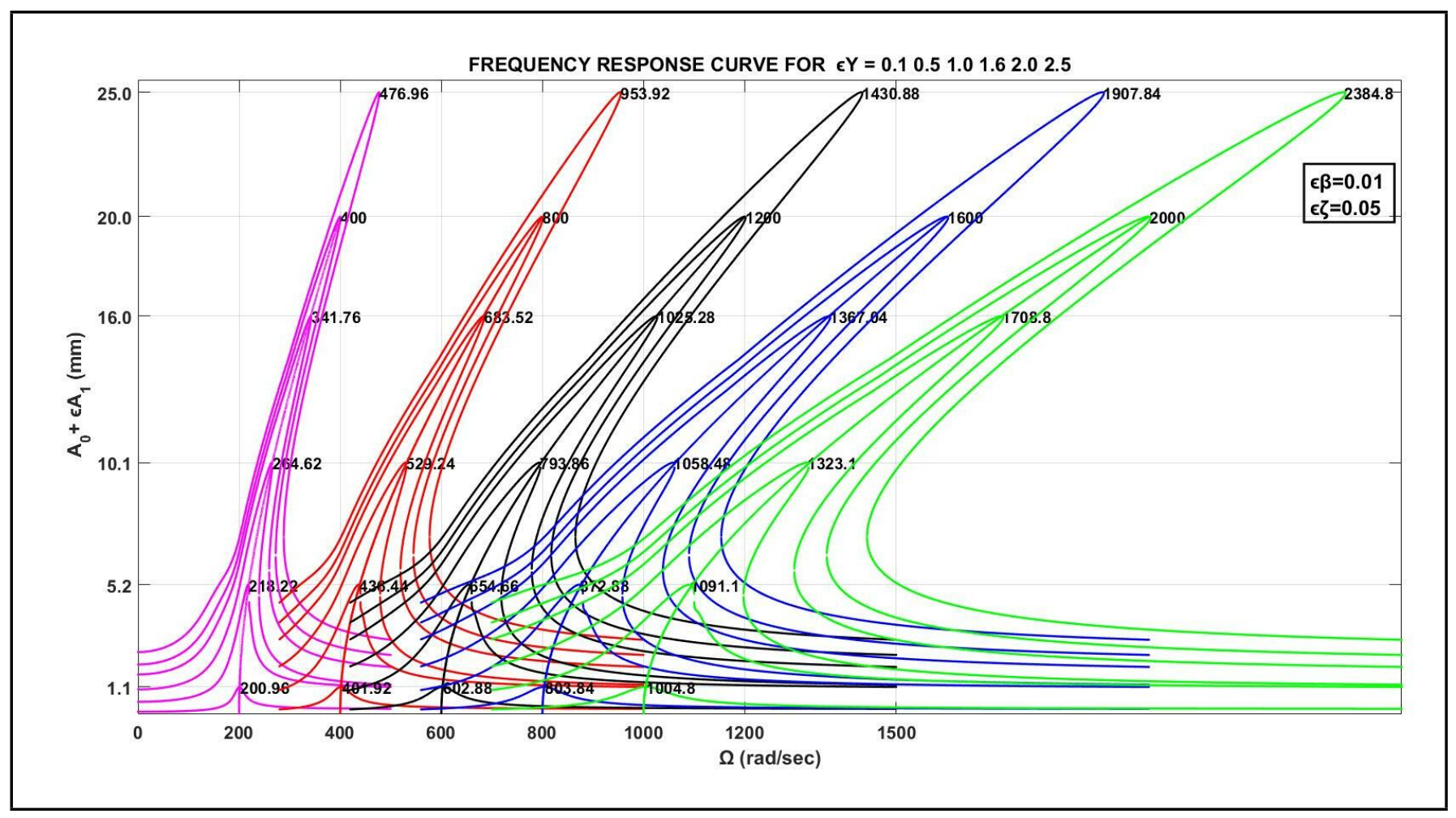

Figure 13. Frequency response $\left(A_{0}+\epsilon A_{1}\right)$ of hard spring $(\epsilon \beta=0.01)$. 


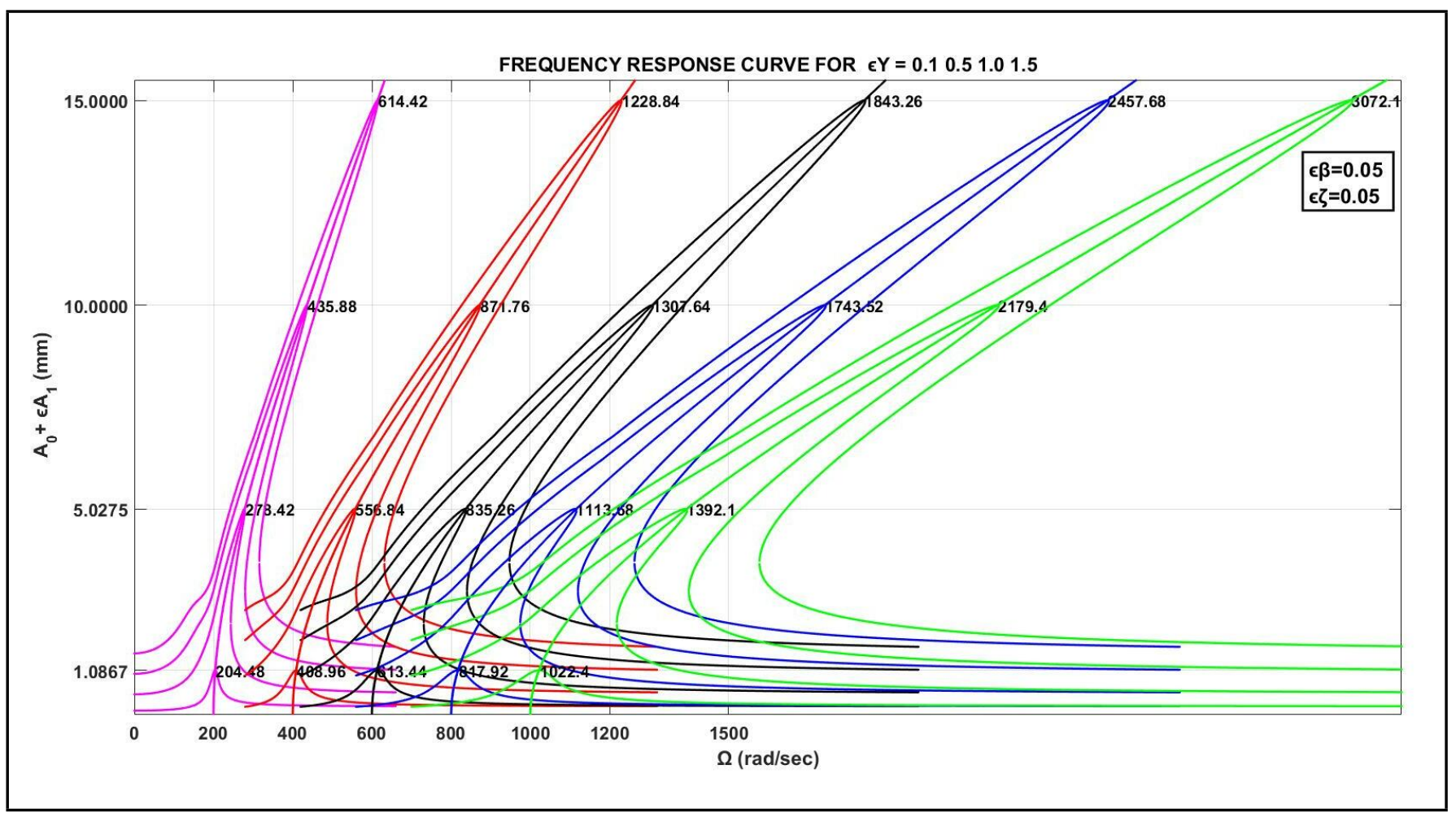

Figure 14. Frequency response $\left(A_{0}+\epsilon A_{1}\right)$ of hard spring $(\epsilon \beta=0.05)$.

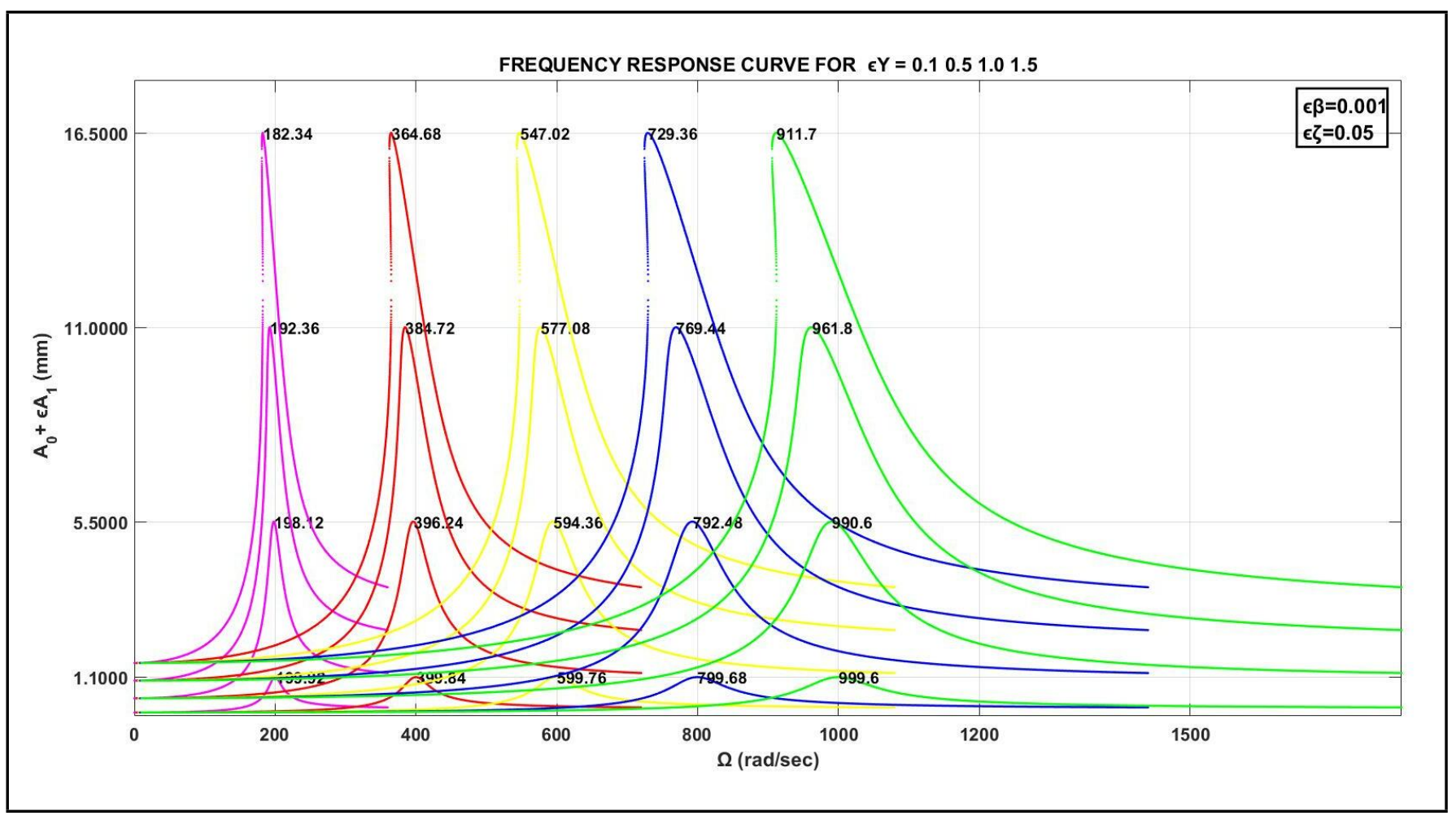

Figure 15. Frequency response $\left(A_{0}+\epsilon A_{1}\right)$ of soft spring $(\epsilon \beta=0.001)$. 


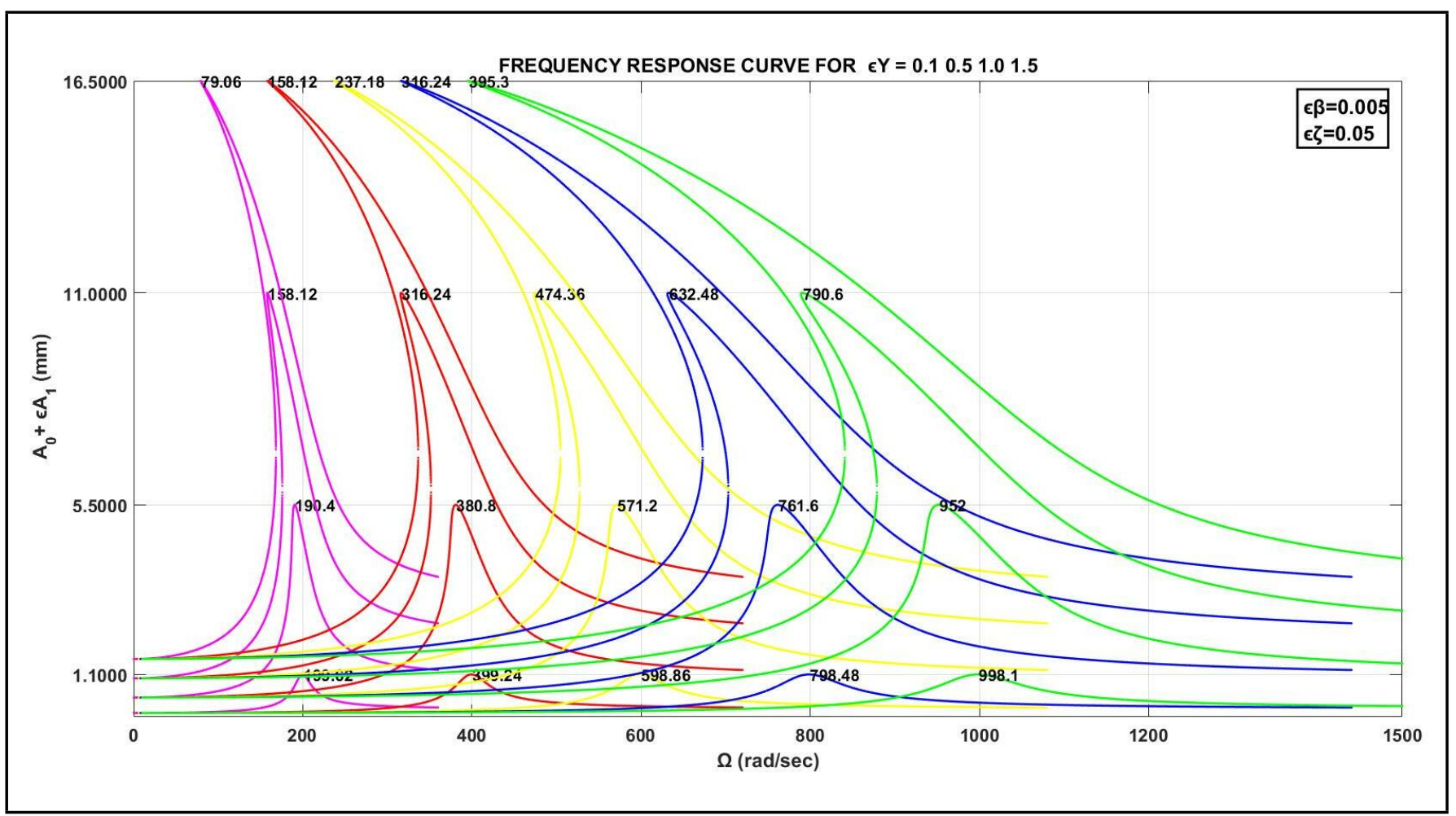

Figure 16. Frequency response $\left(A_{0}+\epsilon A_{1}\right)$ of soft spring $(\epsilon \beta=0.005)$.

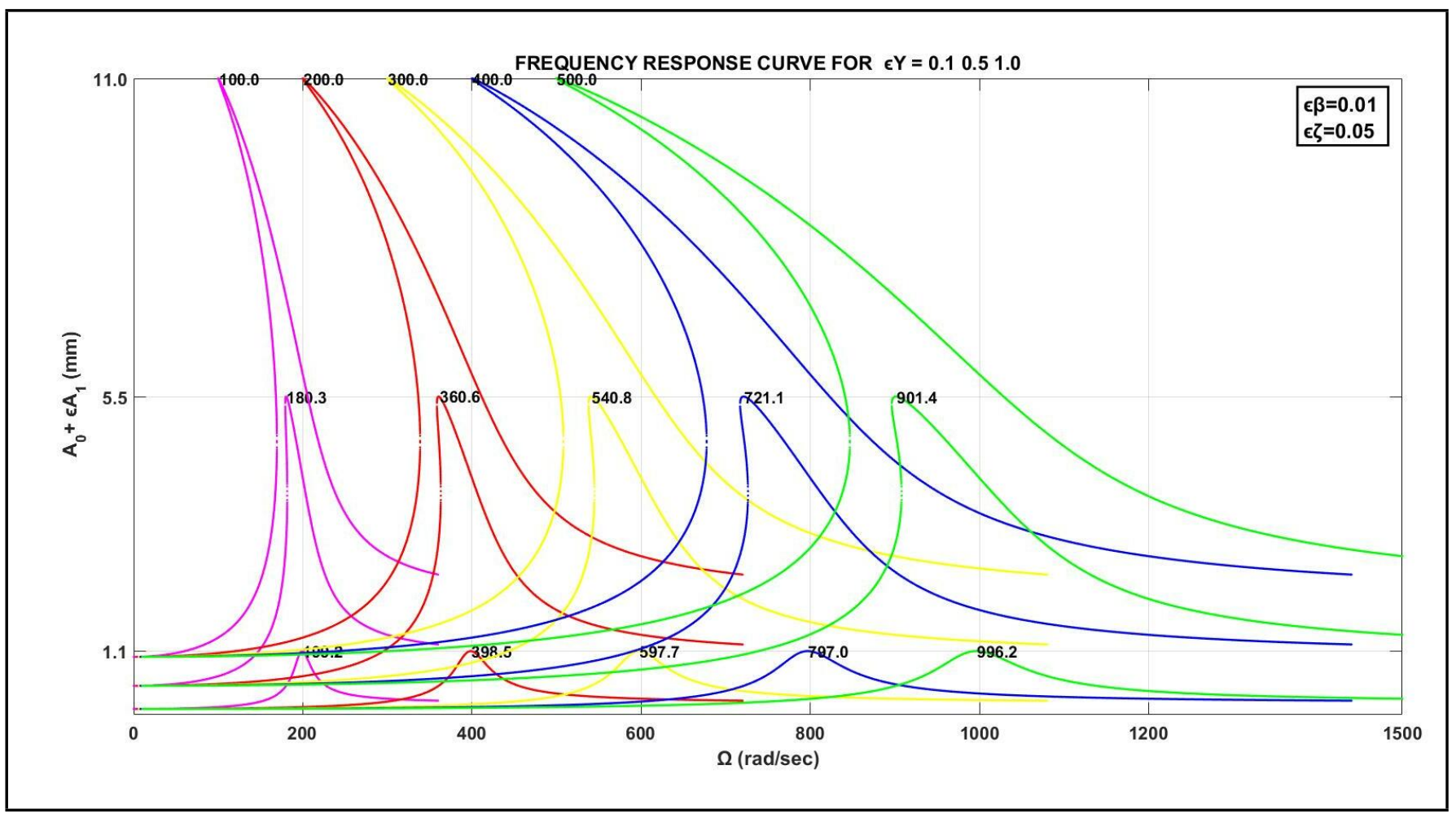

Figure 17. Frequency response $\left(A_{0}+\epsilon A_{1}\right)$ of soft spring $(\epsilon \beta=0.01)$. 


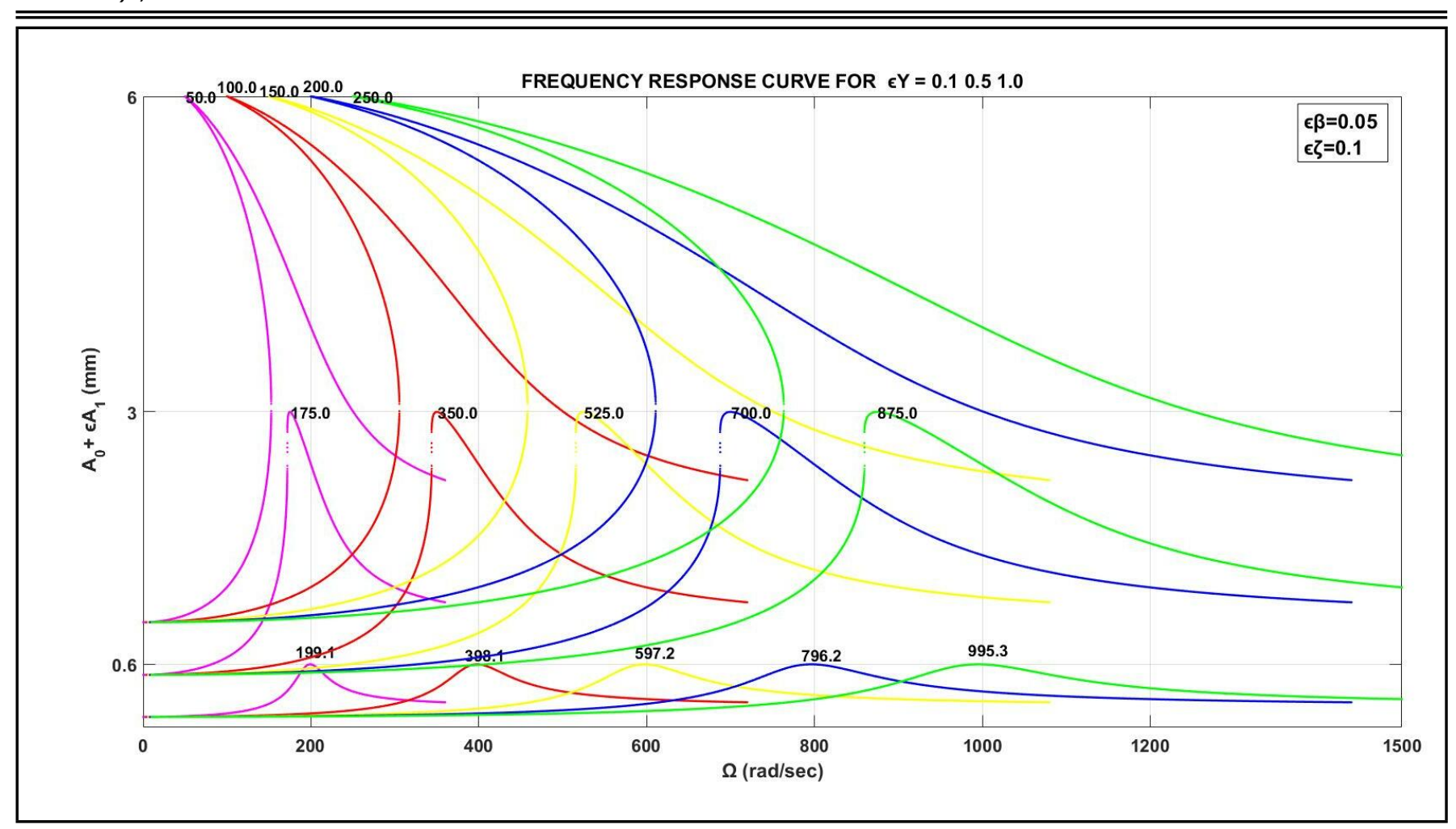

Figure 18. Frequency response $\left(A_{0}+\epsilon A_{1}\right)$ of soft spring $(\epsilon \beta=0.05)$.

\section{REFERENCES}

1 Den Hartog, J. P., Mechanical Vibrations, McGraw-Hill, New York, (1962).

2 Nayfeh, A. H. and Mook, D. T., Nonlinear Oscillations, Wiley, New York, (1979).

3 Vaiana, N., Spizzuoco, M. and Serino, G., Wire rope isolators for seismically base-isolated lightweight structures: experimental characterization and mathematical modeling, Engineering Structures 140 (1), 498-514, (2017). https://dx.doi.org/10.1016/j.engstruct.2017.02.057

4 Sudhir K., Attributes of a vibration isolator design with stiffness nonlinearities, Int. J. of Acoustics and Vibration, 23 (2), 208-216, (2018). https://dx.doi.org/10.20855/ijav.2018.23.21413

5 Losanno, D., Madera Sierra, I. E., Spizzuoco, M., Marulanda, J., and Thomson, P., Experimental assessment and analytical modeling of novel fiber-reinforced isolators in unbounded configuration, Composite Structures 212, 66-82, (2019). https://dx.doi.org/10.1016/j.compstruct.2019.01.026

6 Madera Sierra, I.E., Losanno, D., Strano, S., Marulanda, J. and Thomson, P., Development and experimental behavior of HDR seismic isolators for low-rise residential buildings, Engineering Structures, 183, 894-906, (2019). https://dx.doi.org/10.1016/j.engstruct.2019.01.037

7 Xue X., Wo X., Sun Q. and Zhang L., Simulation of the hysteresis mathematical model for the MR fluid damper using a hybrid evolutionary algorithm, Int. J. of Acoustics and Vibration, 20 (4), 207-219, (2015). https://dx.doi.org/10.20855/ijav.2015.20.4384

8 Nuzzo, I., Losanno, D., Caterino, N., Serino, G. and Bozzo Rotondo, L. M., Experimental and analytical characterization of steel shear links for seismic energy dissipation, Engineering Structures 172, 405-418, (2018). https://dx.doi.org/10.1016/j.engstruct.2018.06.005

9 Nuzzo, I., Losanno, D., and Caterino, N., Seismic design and retrofit of frame structures with hysteretic dampers: a simplified displacement-based procedure, Bulletin of Earthquake Engineering 17 (5), 2787-2819, (2019). https://dx.doi.org/10.1007/s10518-019-00558-8

10 Losanno, D., Spizzuoco, M., and Serino, G., Design and retrofit of multistory frames with elasticdeformable viscous damping braces, Journal of Earthquake Engineering 23 (9), 1441-1464, (2019) https://dx.doi.org/10.1080/13632469.2017.1387193

11 Meirovitch, L., Fundamentals of Vibrations, McGraw-Hill, New York, (2001).

12 Mendelson, K. S., Perturbation Theory for Damped Nonlinear Oscillations, Journal of Mathematical Physics, 11 (12), 3813-3815, (1970). https://dx.doi.org/10.1063/1.1665141

13 Nayfeh, A. H., Perturbation Methods, Wiley, New York, (1973).

14 Friswell, M. I. and Penny, J.E.T., The accuracy of jump frequencies in series solutions of the response of a Duffing oscillator, J. Sound Vib., 169 (2), 261-269, (1994). https://dx.doi.org/10.1006/jsvi.1994.1018 
15 Worden, K., On jump frequencies in the response of the Duffing oscillator, J. Sound Vib., 198 (4), 522-525, (1996). https://dx.doi.org/10.1006/jsvi.1996.0586

16 Malatkar, P. and Nayfeh, A.H., Calculation of the jump frequencies in the response of SDOF non-linear systems, J. Sound Vib., 254 (5), 1005-1011, (2002). https://dx.doi.org/10.1006/jsvi.2001.4104

17 Brennan, M. J., Kovacic, I., Carrella, A. and Waters, T.P., On the jump-up and jump-down frequencies of the Duffing oscillator, J. Sound Vib., 318 (5), 1250-1261, (2008). https://dx.doi.org/10.1016/j.jsv.2008.04.032

18 Gandino, E. and Marchesiello, S., Identification of a Duffing oscillator under different types of excitation, Mathematical problems in Engineering, 1-15, (2010) . https://dx.doi.org/10.1155/2010/695025

19 Bouc, R., Modele mathematique d'hysteresis, Acustica 24, 16-25, (1971).

20 Wen, Y. K., Method for random vibration of hysteretic systems, Journal of the Engineering Mechanics Division, ASCE 102 (2), 249-263, (1976).

${ }^{21}$ Wen, Y. K., Equivalent linearization for hysteretic systems under random excitation, Journal of Applied Mechanics, ASME 47 (1), 150-154, (1980). https://dx.doi.org/10.1115/1.3153594

22 Vaiana, N., Sessa, S., Marmo, F., and Rosati, L., A class of uniaxial phenomenological models for simulating hysteretic phenomena in rate-independent mechanical systems and materials, Nonlinear Dynamics 93 (3), 1647-1669, (2018). https://dx.doi.org/10.1007/s11071-018-4282-2

${ }^{23}$ Vaiana, N., Sessa, S., Marmo, F., and Rosati, L., An accurate and computationally efficient uniaxial phenomenological model for steel and fiber reinforced elastomeric bearings, Composite Structures 211, 196-212, (2019). https://dx.doi.org/10.1016/j.compstruct.2018.12.017

24 Vaiana, N., Sessa, S., Marmo, F., and Rosati, L., Nonlinear dynamic analysis of hysteretic mechanical systems by combining a novel rate-independent model and an explicit time integration method, Nonlinear Dynamics, 98 (4), 2879-2901 (2019). https://dx.doi.org/10.1007/s11071-01905022-5

${ }^{25}$ Hundal, M. S., Response of a base excited system with Coulomb and viscous friction, J. Sound Vib., 64 (3), 371-378, (1979). https://dx.doi.org/10.1016/0022$460 \times(79) 90583-2$

${ }^{26}$ Sun, X., and Zhang, J., Displacement transmissibility characteristics of harmonically base excited damper isolators with mixed viscous damping, Shock and Vibration, 20 (5), 921-931, (2013). https://dx.doi.org/10.3233/SAV-130794
27 EI-Bassiouny, A. F., and Naturforsch, Z. The response of nonlinear single degree-of-freedom systems to modulated high frequency input, Zeitschrift für Naturforschung A, 61 (10-11), 541-555, (2006). https://dx.doi.org/10.1515/zna2006-10-1105

28 Wang, X., Yao, H., and Zheng, G., Enhancing the isolation performance by a nonlinear secondary spring in the Zener model, Nonlinear Dynamics, 87(4), 2483-2495, (2017). https://dx.doi.org/10.1007/s11071-016-3205-3

29 Ravindra, B. and Mallik, A. K., Hard Duffing-Type Vibration Isolator with Combined Coulomb and Viscous Damping, Int. J. Non-Linear Mech., 28 (4), 427-440, (1993). https://dx.doi.org/10.1016/0020-7462(93)90017-F

${ }^{30}$ Ravindra, B. and Mallik, A. K., Performance of Nonlinear Vibration Isolators Under Harmonic Excitation, J. Sound Vib., 170 (3), 325-337, (1994). https://dx.doi.org/10.1006/jsvi.1994.1066

31 Milovanovic, Z., Kovacic, I., and Brennan, M. J., On the Displacement transmissibility of a base excited viscously damped nonlinear vibration isolator, ASME Journal of Vibration and Acoustics, 131 (5), 1-7, (2009). https://dx.doi.org/10.1115/1.3147140

32 Zaghari, B., Rustighi, E., and Tehrani, M. G., Dynamic response of a nonlinear parametrically excited system subject to harmonic base excitation, Journal of Physics: Conference Series, 744 (1),1-10, (2016). https://dx.doi.org/10.1088/1742-6596/744/1/012125

${ }^{33}$ Naik, R. D. and Singru, P. M., Stability of a nonlinear quarter-car system with multiple time-delay, Int. J. of Acoustics and Vibration, 20 (2), 90-94, (2015). https://dx.doi.org/10.20855/ijav.2015.20.2372

${ }^{34}$ Kabir, H., and Sadeghi, M., An optimized method for calculating the linear and nonlinear response of SDOF system subjected to an arbitrary base excitation, International Journal of Civil and Environmental engineering, 11 (3), 380-385, (2017).

35 Greco, F., Luciano, R., Serino, G. and Vaiana, N., A mixed explicit-implicit time integration approach for nonlinear analysis of base-isolated structures. Annals of Solid and Structural Mechanics 10 (1-2), 17-29, (2017). https://dx.doi.org/10.1007/s12356-017-0051-z

${ }^{36}$ Esmailzadeh, E. and Nakhaie-Jazar, G., Periodic behavior of a cantilever beam with end mass subjected to harmonic base excitation, International Journal of Non-Linear mechanics, 33 (4), 567-577, (1998). https://dx.doi.org/10.1016/S0020-7462(97)00038-3

37 Sayag, M. R. and Dowell, E. H., Linear versus nonlinear response of a cantilevered beam under harmonic base excitation: theory and experiment, Journal of Applied Mechanics, 83 (10), 6-13, (2016). https://dx.doi.org/10.1115/1.4034117 